Article

\title{
Impact of Diagenesis on the Reservoir Properties of the Cretaceous Sandstones in the Southern Bredasdorp Basin, Offshore South Africa
}

\author{
Temitope Love Baiyegunhi ${ }^{1, *(1)}$, Kuiwu Liu ${ }^{1}$, Oswald Gwavava ${ }^{1}$ and Christopher Baiyegunhi ${ }^{2}$ \\ 1 Department of Geology, University of Fort Hare, Private Bag X1314, Alice 5700, Eastern Cape Province, \\ South Africa; kliu@ufh.ac.za (K.L.); ogwavava@ufh.ac.za (O.G.) \\ 2 Department of Geology and Mining, University of Limpopo, Private Bag X1106, Sovenga 0727, \\ Limpopo Province, South Africa; christopher.baiyegunhi@ul.ac.za \\ * Correspondence: lovedestiny324@yahoo.com
}

Received: 19 May 2020; Accepted: 8 July 2020; Published: 27 August 2020

\begin{abstract}
The Cretaceous sandstone in the Bredasdorp Basin is an essential potential hydrocarbon reservoir. In spite of its importance as a reservoir, the impact of diagenesis on the reservoir quality of the sandstones is almost unknown. This study is undertaken to investigate the impact of digenesis on reservoir quality as it pertains to oil and gas production in the basin. The diagenetic characterization of the reservoir is based on XRF, XRD SEM + EDX, and petrographic studies of 106 thin sections of sandstones from exploration wells E-AH1, E-AJ1, E-BA1, E-BB1 and E-D3 in the basin. The main diagenetic processes that have affected the reservoir quality of the sandstones are cementation by authigenic clay, carbonate and silica, growth of authigenic glauconite, dissolution of minerals and load compaction. Based on the framework grain-cement relationships, precipitation of the early calcite cement was either accompanied or followed up by the development of partial pore-lining and pore-filling clay cements, particularly illite. This clay acts as pore choking cement, which reduces porosity and permeability of the reservoir rocks. The scattered plots of porosity and permeability versus cement + clays show good inverse correlations, suggesting that the reservoir quality is mainly controlled by cementation and authigenic clays.
\end{abstract}

Keywords: diagenesis; reservoir quality; porosity; sandstones; Bredasdorp Basin

\section{Introduction}

Reservoir quality is one of the main controls on prospectivity during petroleum exploration. During petroleum exploration, remote sensing techniques and geophysical methods are useful in identifying the presence of sandstones. However, they do not give any information or help to locate sandstones with high porosity and permeability that have been preserved from major diagenetic changes and porosity-loss [1,2]. Hence, it is very crucial to have a thorough understanding of the factors controlling reservoir quality so as to assist with the appraisal of the economic feasibility of petroleum discoveries. Reservoir quality of fine-grained clastic rocks is affected by different interrelated factors that includes pore water chemistry, mineral composition, temperature, diagenetic events, subsurface pressure, burial depth and time of uplift, depositional environment and tectonic setting [1-3]. Several researchers including [3-7] have reported that diagenetic changes or modifications in clastic rocks have a momentous impact on reservoir quality by altering their original porosity and permeability, which in turns affects the reservoir quality. A thin section petrography is a crucial and important tool used for investigating the types and timing at which the diagenetic processes have affected porosity and permeability of clastic rocks [8]. Also, the economic importance of certain sandstone units as 
a source or reservoir rock for petroleum depends on the diagenetic history of the units as well as their original depositional characteristics [5,9]. There are a vast number of studies which have drawn these conclusions in the past [3,10-13], and many of the reactions and diagenetic alterations presented in this study in particular are quite well understood. However, diagenetic modifications in reservoir properties of the Bredasdorp Basin is still not reported or documented to date.

The Bredasdorp Basin covers the greater part of the larger Outeniqua Basin representing a productive petroleum bearing basin, off the south coast of South Africa (Figure 1). Over the past decade, substantial amount of research works has been focused on the geology and petrophysical characterization of the basin due to its hydrocarbon potential. Significant contributions have been made by [14-26] towards the understanding of the regional geology, petroleum prospects, and tectonic evolution of the Bredasdorp Basin, particularly in the northern and central part of the basin. However, up to date, only a few unpublished company's reports and $\mathrm{MSc} / \mathrm{PhD}$ theses exists on the reservoir properties of the Cretaceous sandstones of the Bredasdorp Basin. Incidentally, there still exists a gap between the understanding the reservoir properties and factors that controls the reservoir quality of the Bredasdorp sandstones. The purpose of this research work is to investigate the diagenetic characteristics and reservoir quality of the Bredasdorp sandstones in the southern part of the basin so as to close the gap between our understanding of diagenesis and its effects on the reservoir properties.

\section{Geological Setting}

The continental margin extending along the south coast of South Africa developed during the Mesozoic as a result of the break-up or separations of the South American plate from the African-Indian plate, and the African-Indian plate from the Antarctica [27]. During this break-up, the continental margin (passive margin) underwent several tectonic events (rift and drift stages) resulting in the formation of the large Outeniqua Basin as well as its sub-basins. The Bredasdorp Basin is one of five sub-basins of the large Outeniqua Basin, the others are Pletmos, Gamtoos, Algoa and the Southern Outeniqua, covering an area of about $20,000 \mathrm{~km}^{2}, 3000 \mathrm{~km}^{2}, 4000 \mathrm{~km}^{2}$ and 22,000 $\mathrm{km}^{2}$, respectively [20]. The large Outeniqua Basin developed along the south coast of South Africa, in response to rift tectonics during the break-up of Gondwana [28]. According to [25], the dextral trans-tensional stress or right-lateral shear movement that was produced along the Falkland-Agulhas Fracture Zone (AFFZ) occurred as a result of the separation of the Falkland Plateau from the Mozambique Ridge as well as the break-up of west Gondwana. The tectonic events initiated the development of normal faulting north of the AFFZ, resulting in the creation of graben and half-graben sub-basins including the Bredasdorp Basin [15]. The Bredasdorp Basin is a south-easterly trending rift basin that is characterized by half-graben structures. These half-grabens are mostly made up of the Upper Jurassic, Lower Cretaceous, Cretaceous and Cenozoic rift to drift strata.

The Bredasdorp Basin is the focus of this study and it developed along the South African continental margin, underneath the Indian Ocean during late Jurassic-early Cretaceous in response to the extensional episodes during the early stages of rifting. Geographically, the basin is located off the south coast of South Africa (south-east of Cape Town and west-south-west of Port Elizabeth) and covers an area extent of about $18,000 \mathrm{~km}^{2}$ beneath the Indian Ocean along the southern coast of South Africa [19]. The Bredasdorp Basin is about $200 \mathrm{~m}$ long and $80 \mathrm{~km}$ wide [20,24] and it is bounded on the western and southwestern side by the Columbine-Agulhas Arch and on the northeastern side by the Infanta Arch [15]. The Infanta Arch physically separates the Bredasdorp Basin from the Pletmos Basin. These arches are elongated basement highs and they are made up of the Cape Supergroup metasediments, granite and Precambrian (basement) metamorphic rocks. Geologically, the tectono-sedimentary history of the Bredasdorp Basin can be dated back to the Permo-Triassic Cape Orogeny. Most of the structural features of the basin (i.e., faults, horst and grabens) show a strong parallelism with the regional structural trend of the Swartberg Branch of the Cape Fold Belt [29].

Stratigraphically, the Bredasdorp Basin hosts an Oxfordian-Recent stratigraphic column [22] overlying the Cape Supergroup [17]. The stratigraphic column in Figure 2 shows the syn-rift phase 
(late Jurassic-early Cretaceous), post-rift or drift phase (early Cretaceous-Tertiary), and the duration of stratigraphic events (deposition versus hiatus) in a Wheeler-type stratigraphic diagram [16]. The rift phase is characterized by extension-driven subsidence and synrift basin fill. Isostatic uplift of both flanks of the half graben led to the erosional truncation of the late-rift sediments, whereas extreme marginal uplift and erosion of the northern flank removed the whole synrift sequence in places [20]. The rift phase can be subdivided into the synrift and post-rift sequence. The synrift sedimentary sequence is made up of non-marine (fluvial) and shallow marine sediments, while the post-rift sequence consists of deep marine sediments [30]. The drift succession refers to the sets of stratigraphic units and their deposition has been linked to the initial and final period of the gradual west-southwest-ward plates of southern coast of Africa. These movements led to the formation of the Outeniqua sub-basins. The Early drift phase occurred just before the onset of rapid thermal subsidence and it is associated with continued uplift, resulting in erosion truncation on the southern edges. Rapid subsidence and deposition of deep water sediments took place within the graben [20]. The drift phase is characterized by a regional subsidence that was compelled by thermal cooling and sediment loading with continued minor movement on the Arniston fault. Continued subsidence (minor) up to the Early Tertiary when alkaline intrusive affected the southern flank as well as late slanting of the basin, led to uplift and erosion [20]. The structural development of the Bredasdorp Basin is summarized in Table 1.

According to [14], exploratory drilling in the basin discovered gas-condensate and oil reservoirs. However, uncertainty concerning the depth and maturity of the source kitchen hinders further exploration. Also, thermal history of the basin is complex due to the unusual sequence of heating events. As reported by [31], post-rift subsidence in the basin as a result of reduced heat flow, near to present levels, bring about a reduction in the temperature gradients through to the late Cretaceous. During the latest Cretaceous and early Tertiary, Africa migrated over a mantle plume directly related to a hotspot, and regional uplift and heating ensued [32,33]. The path of the hotspot is indicated by [32] to traverse the area of the Bredasdorp Basin during late Cretaceous. Also, the Southern Outeniqua Basin is adjacent to the highly thermally conductive Agulhas fracture ridge at the edge of the continental plate [34]. Since the emergence of the ridge, quick heat transfers from the base of the lithosphere through this ridge and in recent times, from the emerging mantle swell and plume-head [35], could have resulted in greater palaeo- and present geothermal gradients than in the Bredasdorp Basin [31]. Also, thicknesses of the sediment locally surpass $9 \mathrm{~km}$ [36], hence pore fluids are likely to be meaningfully hotter than pore fluids in the Bredasdorp Basin, reaching $>250^{\circ} \mathrm{C}$ [31]. The present temperature gradients in the Bredasdorp Basin are high, ranging between $35-49^{\circ} \mathrm{C} \mathrm{km}^{-1}$ [37] and indicate a moderate-high present day heat flow, averaging $53 \mathrm{~mW} \mathrm{~m}^{-2}$ [31]. Sonibare [38] reported that the paleo-water depths in the basin remain relatively shallow over the past and up to present-day, varying from about $40 \mathrm{~m}$ up to $300 \mathrm{~m}$ with maximum water-depths occurring during Hauterivian-Aptian times. According to [31], the source rock quality in the basin is wet gas, wet gas to oil and oil prone (Table 1).

The thermal maturity of dispersed organic matter partly controls the behaviour of the organic matter and consequently may influence the interpretation of hydrocarbon generation. Therefore, when assessing or evaluating hydrocarbon generation, it is very important to be mindful of the effects of maturation on the organic matter and to take this effect into consideration. Vitrinite reflectance is the most suitable and widely accepted by several researchers and exploration geologists as a method for measuring the thermal maturity of source rocks. According to [39], rocks with vitrinite reflectance between $0.20-0.60 \%, 0.60-1.35 \%$ and above $1.35 \%$ are termed immature, mature and post-mature, respectively. In terms of the source area, McCarthy et al. [40] reported that the Type-I kerogen is mostly derived from algae and associated with lacustrine depositional settings. The Type II kerogen is chiefly derived from plankton, with some contribution from algal material, hence indicating a marine depositional setting. furthermore, marine environments with substantial terrestrial input, are alleged to be the depositional environments for the Type III kerogen, whereas the Type IV kerogen is mostly derived from reworked organic matter (mainly dead carbon). The geochemical parameters for describing kerogen type, hydrocarbon type and organic carbon richness are presented in Table 2. 
Hydrogen Index (HI) is the amount of hydrogen contained within the kerogen, with higher HI indicating a potential for oil production. The hydrocarbon generation phase and the burial history of the basin is shown in Figure 3. According to [41], hydrocarbons generation in the basin took place as result of burial depth that had been reached in the early Tertiary (60 Ma) with a next phase of hydrocarbon generation in the late Tertiary $(5 \mathrm{Ma})$. There is a possibility that other source rocks were mature for oil generation before the end of the early Cretaceous [41]. As earlier indicated, the geothermal gradient in the basin is relatively high, resulting in most of the Lower Cretaceous source rocks being in the gas window. However, in the deeper parts of the basin, the Upper Cretaceous source rocks is likely to be mature and in the oil window (Table 1).

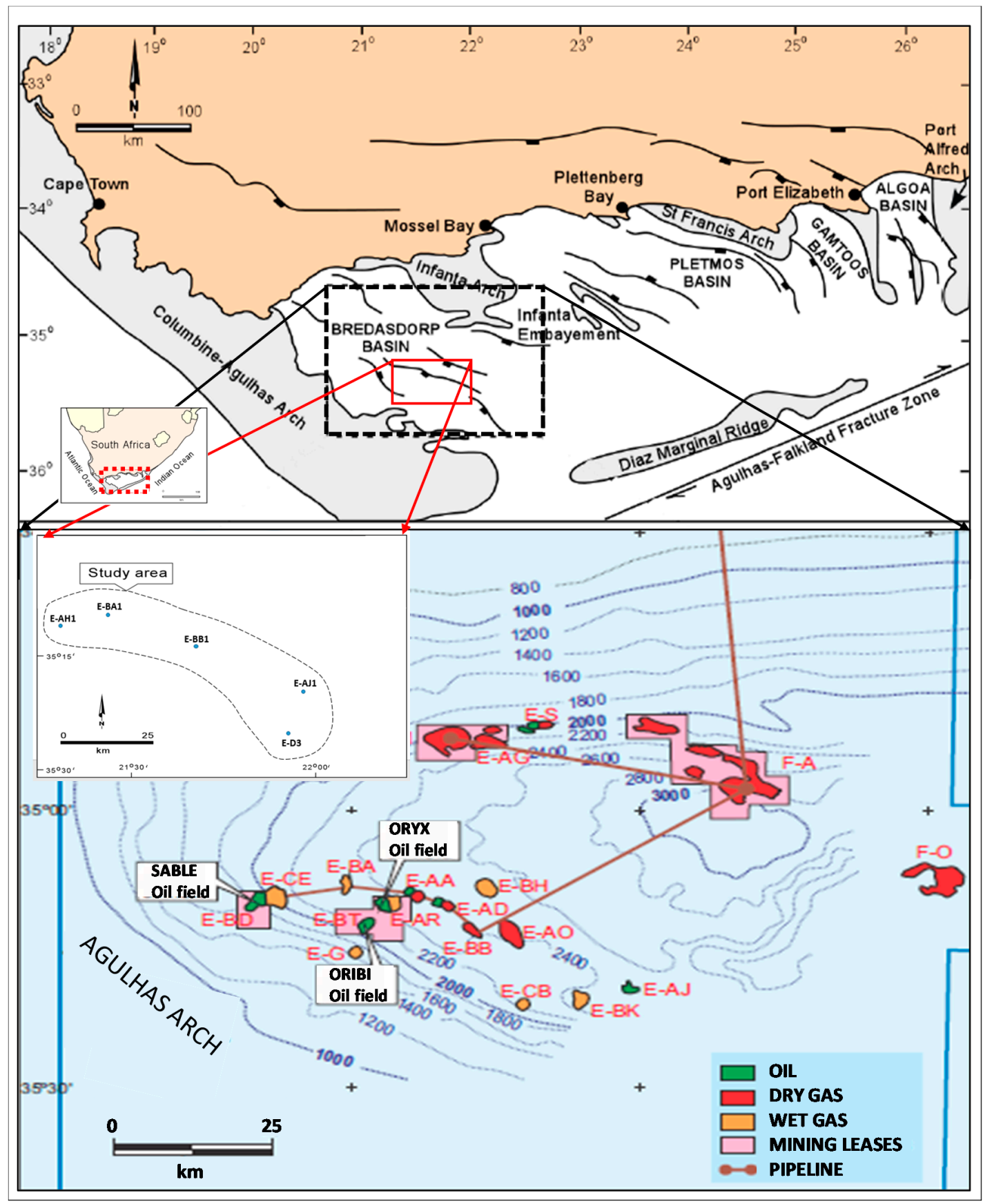

Figure 1. Map of the study area showing distribution of the exploration wells across the Bredasdorp Basin (modified from [42]). 


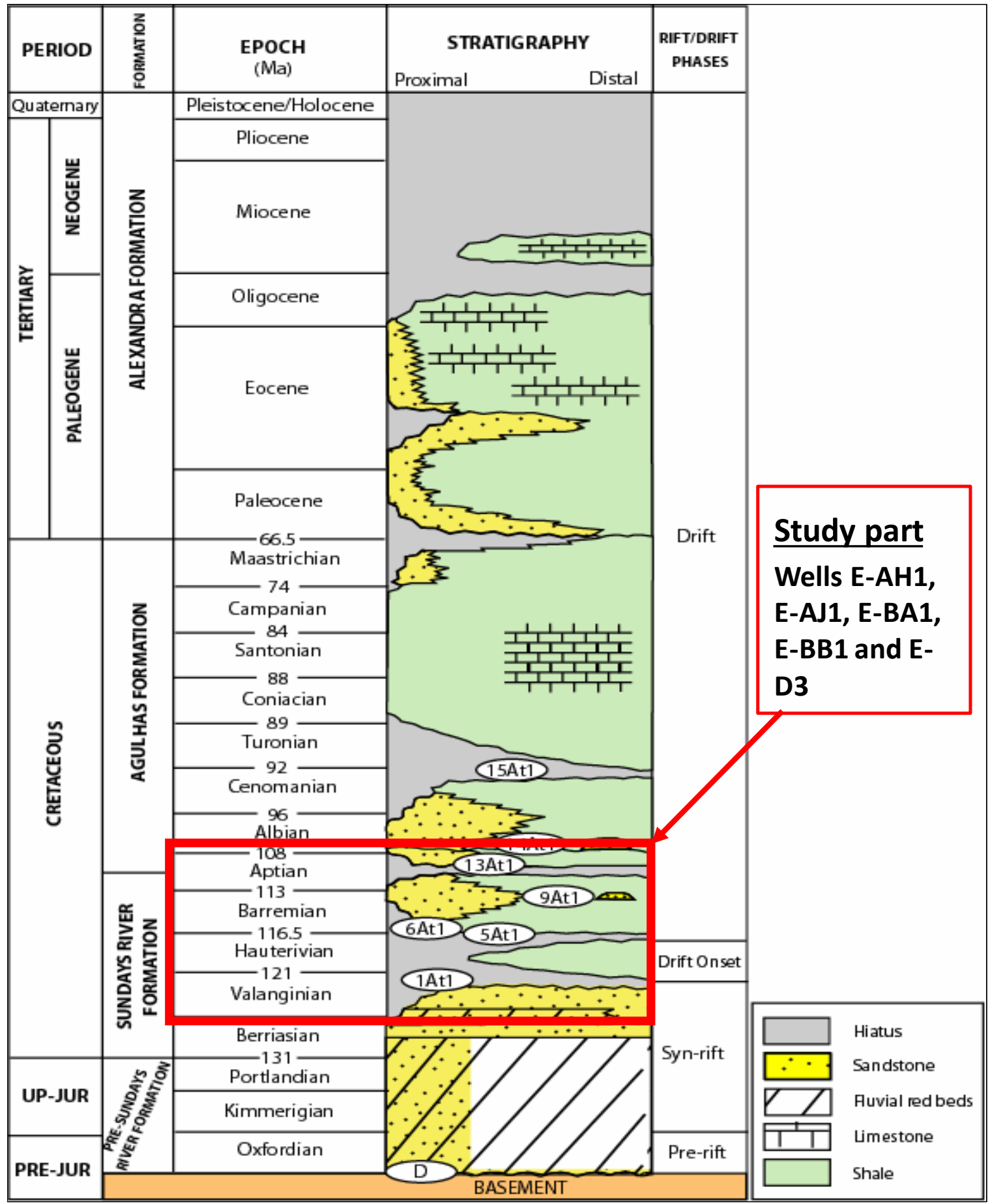

Figure 2. Sequence chronostratigraphy of the Bredasdorp Basin (after [38]). 
Table 1. Summary of the structural development of the Bredasdorp Basin hydrocarbon potential and maturity of source rocks (after [21,43]).

\begin{tabular}{|c|c|c|c|c|c|}
\hline Stage & Period & Phase & Major Event & $\begin{array}{l}\text { Hydrocarbon } \\
\text { Potential }\end{array}$ & $\begin{array}{c}\text { Maturity } \\
\text { (Ro\%) }\end{array}$ \\
\hline 1 & $\begin{array}{l}\text { middle Jurassic to } \\
\text { Valanginian } \\
\text { (Basement to 1At1) }\end{array}$ & Synrift I & $\begin{array}{l}\text { Extension-instigated or driven } \\
\text { subsidence and syndrift basin } \\
\text { fill. Isostatic uplift on both } \\
\text { side of the half-graben led to } \\
\text { significant reduction in the } \\
\text { erosion of Synrift I sediments. } \\
\text { Great or intensive marginal } \\
\text { uplift and erosion of the } \\
\text { northern part of the basin } \\
\text { removed the whole Synrift I } \\
\text { succession in some places }\end{array}$ & $\begin{array}{l}\text { Dry Gas Or Oil } \\
\text { and non- } \\
\text { hydrocarbons }\end{array}$ & $1.0-1.5 \%$ \\
\hline 2 & $\begin{array}{l}\text { late Valanginian to } \\
\text { Hauterivian } \\
\text { (1At1 to 6At1) }\end{array}$ & Synrift II & $\begin{array}{l}\text { Fast subsidence and extensive } \\
\text { flooding. Continued or } \\
\text { non-stop uplift caused } \\
\text { additional truncation or } \\
\text { reduction of structural highs. } \\
\text { The deposition of deepwater } \\
\text { sediments happened within } \\
\text { rift depocentres (Southern } \\
\text { Sub-Basin and Arniston } \\
\text { Half-graben) led to the } \\
\text { deposition of source rocks }\end{array}$ & Gas & $0.9-1.5 \%$ \\
\hline 3 & $\begin{array}{l}\text { Hauterivian to Aptian } \\
\text { (6At1 to 13At1) }\end{array}$ & $\begin{array}{l}\text { Transitional } \\
\text { (Early Drift) }\end{array}$ & $\begin{array}{l}\text { Progradation enlargement of } \\
\text { the shelf in the northern part } \\
\text { over the Arniston Half-graben, } \\
\text { plus a sustained deepening of } \\
\text { the Southern Sub-Basin }\end{array}$ & Wet Gas-Oil & $0.8-1.3 \%$ \\
\hline 4 & $\begin{array}{l}\text { Aptian to Maastrichtian } \\
\text { (13At1 to 15At1) }\end{array}$ & Middle Drift & $\begin{array}{c}\text { Regional subsidence } \\
\text { instigated by thermal cooling } \\
\text { and sediment loading. } \\
\text { Continued movement on the } \\
\text { Arniston Fault }\end{array}$ & Oil & $0.7-1.2 \%$ \\
\hline 5 & $\begin{array}{l}\text { Paleocene to Present Day } \\
\quad \text { (15At1 to Seafloor) }\end{array}$ & Late Drift & $\begin{array}{l}\text { Oil-prone source in synrift } \\
\text { depocentres and northern and } \\
\text { central part of the Bredasdorp } \\
\text { Basin proceed into the main } \\
\text { phase of oil generation. } \\
\text { Continued slight subsidence } \\
\text { interrupted by the early } \\
\text { Tertiary alkaline intrusion } \\
\text { activity over the Central part } \\
\text { of the basin. Late slanting of } \\
\text { the basin together with uplift } \\
\text { of the northern side led to the } \\
\text { late removal or erosion of } \\
\text { about } 600 \text { m succession in } \\
\text { some places }\end{array}$ & Oil & $0.5-0.7 \%$ \\
\hline
\end{tabular}


Table 2. Geochemical parameters for describing the kerogen type and hydrocarbon type generated [16].

\begin{tabular}{ccc}
\hline Kerogen Type & $\begin{array}{c}\text { Hydrogen Index } \\
(\mathbf{m g H C} / \mathbf{g} \text { TOC })\end{array}$ & Main Expelled at Peak Maturity \\
\hline I & $>600$ & Oil \\
\hline II & $300-600$ & Oil \\
\hline II/III & $200-300$ & Mixed oil and gas \\
\hline III & $50-200$ & Gas \\
\hline IV & $<50$ & Dry Gas \\
\hline
\end{tabular}

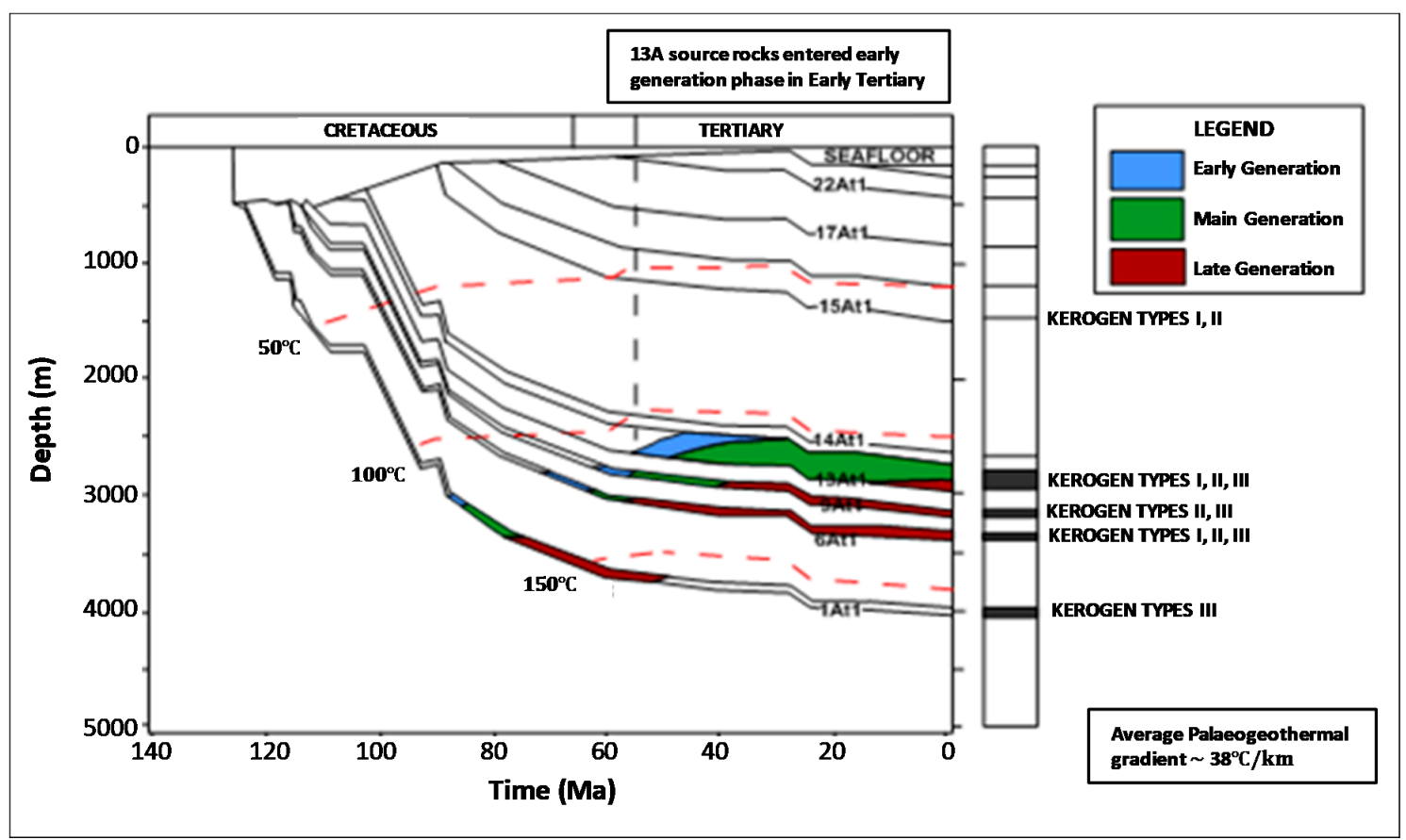

Figure 3. Schematic diagram showing Burial history graph for Bredasdorp Basin [41].

\section{Materials and Methods}

A total of 40 representative sandstone samples collected from exploration wells E-AH1, E-AJ1, E-BA1, E-BB1 and E-D3 (Figure 1; Table 3) were subjected to X-ray fluorescence (XRF), petrographic microscopy, modal composition, $\mathrm{X}$-ray diffraction (XRD) and scanning electron microscopy (SEM) analyses. The XRF analysis was carried out at the University of Limpopo (Mankweng, South Africa), while SEM analysis was performed at the University of Fort Hare (Alice, South Africa). For the XRF, the samples were milled to less than $50 \mu \mathrm{m}$ grain size and pressed pellets were prepared. The pressed pellets were analysed for the major oxides using a PANalytical Zetium XRF spectrometer equipped with a $4 \mathrm{~kW}$ Rh tube. The milled samples were also dried at $100^{\circ} \mathrm{C}$ and heated at $1000{ }^{\circ} \mathrm{C}$ for a period of at least $3 \mathrm{~h}$ to oxidize sulphur (S) and iron $\left(\mathrm{Fe}^{2+}\right)$ in order to determine the loss of ignition (LOI). Thin sections petrographic study and modal composition analysis of the sandstones were carried out to determine their mineralogical compositions, textural characteristics and cement types. For the modal composition analysis, at least 500 points were counted per thin section using the procedures recommended by $[44,45]$ which is in accordance with the Gazzi-Dickinson's point-counting technique. The framework components of the sandstones of the Bredasdorp Basin were categorized into quartz (monocrystalline and polycrystalline), feldspar, lithic fragments, mica, cement (i.e., authigenic clay, carbonate and silica) and matrix. The amount of pore spaces was also accounted for during point counting. Thin section visual porosity (point counting) was determined using an Olympus BX51 petrographic microscope 
equipped with an Olympus DP72 digital camera and the results were statistically correlated or compared to the existing gas expansion porosity and Klinkenberg corrected permeability values for the studied exploration wells. Gas expansion porosity and Klinkenberg corrected permeability data used were measured from core plugs by the Petroleum Agency South Africa (PASA, Cape Town, South Africa). Gas expansion porosity and Klinkenberg corrected permeability have been used in all cases. The standard unit of measurement is the American Petroleum Institute (API). To determine the porosity using the gas expansion method, the pore volume was measured by allowing the gas which fills the pores to expand into a measured volume and measuring the accompanying fall in pressure (The method depends on the ideal gas law or Boyle's law). The poroperm instrument is a combined permeameter and porosimeter was used to determine porosity and permeability of plug sized cores at ambient confining pressure. The Klinkenberg correction is a procedure for calibration of permeability data obtained from a minipermeameter device. Herein, the porosity and permeability determined using the poroperm instrument is referred to as gas expansion porosity and Klinkenberg correction permeability. These porosity and permeability values were supplied by the Petroleum Agency of South Africa (extracted from the Soekor's database) and it was incorporated into this study. In addition, 30 sandstone samples were also analysed by X-ray diffraction (XRD) to determine the mineral compositions. The XRD analysis was carried out using the backloading preparation method. The diffractograms were obtained by means of a Malvern PANalytical Aeris diffractometer with a PIXcel detector and fixed slits with Fe-filtered $\mathrm{Co}-\mathrm{K} \alpha$ radiation. The phases were identified using X'Pert Highscore plus software (version 2.1, PANalytical, Malvern, UK), while the relative phase amounts (weight\%) were estimated using the Rietveld method [46]. Furthermore, clay minerals, quartz overgrowth, diagenetic textures, dissolution effect and other related diagenetic imprints were examined using SEM. For the SEM, the sandstones were cleaned, gold coated and analysed using a scanning electron microscopy (SEM) instrument (Model: JEOL JSM-6390LV) fitted with an energy dispersive $x$-ray micro-analyser (EDX). The coated samples were observed in backscattered electron (BSE) and secondary electron imaging (SEI) modes of imaging. The results obtained from SEM and petrographic analyses were used to interpret or comment on the porosity and permeability values. The stratigraphic sections of the studied boreholes are presented in the Supplementary Data.

Table 3. Locations and total drilling depth of the studied exploration wells.

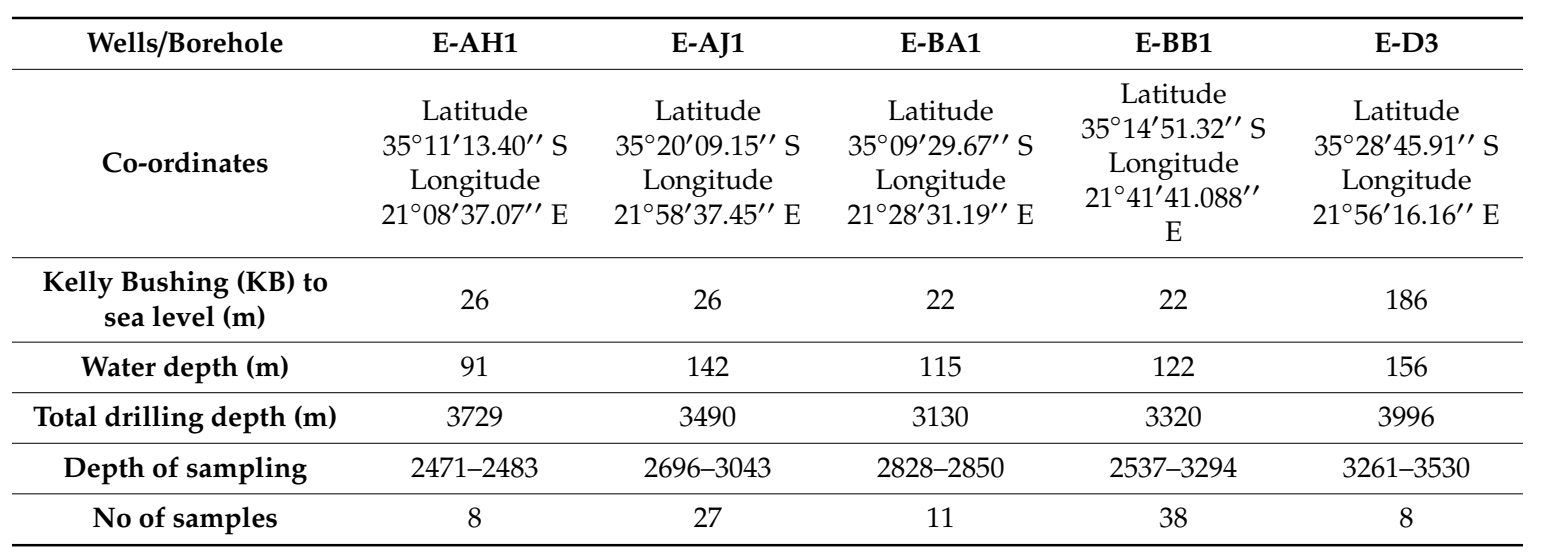

\section{Results}

\subsection{Major Oxides}

The major oxides concentrations in the Bredasdorp sandstones are presented in Table 4. The major oxide compositions are relatively variable, with a general high percentage of $\mathrm{SiO}_{2}$, varying between $51.70 \%$ and $81.58 \%$. The concentration of $\mathrm{Al}_{2} \mathrm{O}_{3}, \mathrm{CaO}, \mathrm{Fe}_{2} \mathrm{O}_{3}, \mathrm{MgO}$, and $\mathrm{K}_{2} \mathrm{O}$ ranges from $2.82 \%$ to $29.01 \%, 0.80 \%$ to $7.68 \%, 0.27 \%$ to $5.75 \%, 0.39 \%$ to $5.68 \%$, and $0.43 \%$ to $5.11 \%$, respectively. 
The percentages of $\mathrm{MnO}, \mathrm{TiO}_{2}, \mathrm{Na}_{2} \mathrm{O}$, and $\mathrm{P}_{2} \mathrm{O}_{5}$ are generally low, ranging from $0.01 \%$ to $0.10 \%, 0.16 \%$ to $0.99 \%, 0.03 \%$ to $1.30 \%$, and $0.11 \%$ to $1.37 \%$, respectively.

\subsection{Modal Composition}

The Bredasdorp sandstones are largely fine to medium grained and moderately sorted to moderately well sorted. The sandstones are made up of framework grains, accessory minerals, matrix, cement and pores. The framework minerals are quartz, feldspar, lithic fragments and glauconite, while the accessory minerals are mica (biotite and muscovite), zircon, and rutile. The quartz grains are usually subangular to rounded in shape and constitute about $52.2-68.0 \%$ of the framework grains (Table 5). The feldspar grains often range from subangular to subrounded shape and constitute nearly $10.0-18.0 \%$ of the framework grains. The lithic fragments constitute about $5.0-10.2 \%$, averaging $7.8 \%$ of the framework grains. These lithic fragments often occur as fine quartz grains in clay matrix (sedimentary lithic fragments) and clasts of sutured grains with no matrix (metamorphic lithic fragments). The sedimentary lithic fragments (fragments of sandstone and mudrock) constitute a larger percentage of the total lithic fragments. The matrix and cement serve as binding materials around larger or framework grains. The matrix or groundmass is mostly clay minerals, constituting about $3.2-13.8 \%$, whereas the cement constitutes between 1.8 and $10.0 \%$ of the overall composition.

\subsection{Mineralogy of the Bredasdorp Sandstones}

The XRD analysis shows that the most abundant minerals include quartz (56.0-73.7\%) and plagioclase (6.1-14.5\%), while the dominant clay mineral is kaolinite (1.6-13.6\%) (Table 6). The observed clay minerals are kaolinite, illite, smectite and chlorite. The XRD patterns are presented in the Supplementary Data.

\subsection{Diagenesis of the Bredasdorp Sandstones}

The primary diagenetic processes that have sturdily affected the reservoir properties of the sandstones are cementation by authigenic clays (i.e., kaolinite, smectite, illite and chlorite), carbonate and silica, growth of authigenic glauconite, dissolution of minerals and load compaction (Figures 4 and 5). Kaolinite in the sandstones is observed under SEM as fibrous or booklets and vermicular aggregates in mud matrix (Figure 4a,b). Smectite occurs as microcrystalline matrix aggregates and grain lining or coating cement (Figure 4c), whereas illite is seen as pore filling and pore lining of cement and sometimes they are seen growing from the surfaces of the curved flake smectite, forming a mixed illite-smectite interlayers (Figure 4e,f). The pyrite is seen in the form framboidal and poikilotopic pyrite cements (Figure 4d). Silica cement occurs in the sandstones as authigenic quartz in pore spaces and as euhedral syntaxial form around quartz grains as overgrowths (Figure 4e,k). Chlorite is seen in the sandstones as pore-filling rosette-like aggregates (Figure 4e). Petrographic and SEM analyses have revealed that the main mineral replacements in the sandstones are the replacement of feldspar with glauconite, replacement of clay matrix and feldspar with calcite, replacement of microcline with albite (albitization), and replacement of one clay minerals with another clay mineral (i.e., kaolinitization and illitization) (Figure 4i,j,l and Figure 5a). Dissolution is a common diagenetic process in the sandstones and it encompasses the removal in solution of part or all of the previously existing minerals, creating secondary pores in the rocks (Figure $5 b$ ). The sandstones show variable degrees of mechanical compaction and little or smaller extent of chemical compaction. The pattern of the grain to grain contact progressively changed from non-contact to point contact, to long contact, then to concavo-convex and sutured contacts (Figure 6). 
Table 4. Results of oxides (wt $\%$ ) analysed by X-ray fluorescence spectrometry.

\begin{tabular}{|c|c|c|c|c|c|c|c|c|c|c|c|c|c|c|c|c|}
\hline Borehole & Depth (m) & Lithology & $\begin{array}{c}\mathrm{SiO}_{2} \\
(\%)\end{array}$ & $\begin{array}{c}\mathrm{TiO}_{2} \\
(\%)\end{array}$ & $\begin{array}{c}\mathrm{Al}_{2} \mathrm{O}_{3} \\
(\%)\end{array}$ & $\begin{array}{c}\mathrm{Fe}_{2} \mathrm{O}_{3} \\
(\%)\end{array}$ & $\begin{array}{c}\mathrm{MnO} \\
(\%)\end{array}$ & $\begin{array}{c}\mathrm{MgO} \\
(\%)\end{array}$ & $\begin{array}{l}\mathrm{CaO} \\
(\%)\end{array}$ & $\begin{array}{c}\mathrm{Na}_{2} \mathrm{O} \\
(\%)\end{array}$ & $\begin{array}{c}\mathrm{K}_{2} \mathrm{O} \\
(\%)\end{array}$ & $\begin{array}{c}\mathrm{P}_{2} \mathrm{O}_{5} \\
(\%)\end{array}$ & $\begin{array}{c}\mathrm{Cr}_{2} \mathrm{O}_{3} \\
(\%)\end{array}$ & $\begin{array}{l}\text { LOI } \\
(\%)\end{array}$ & $\begin{array}{c}\mathrm{H}_{2} \mathrm{O}^{-} \\
(\%)\end{array}$ & $\begin{array}{c}\text { Total } \\
(\%)\end{array}$ \\
\hline E-AH1 & 2471.08 & Sandstone & 51.70 & 0.82 & 28.92 & 4.69 & 0.06 & 1.02 & 1.05 & 0.53 & 2.96 & 0.11 & 0.07 & 7.33 & 0.70 & 99.96 \\
\hline E-AH1 & 2473.98 & Sandstone & 51.26 & 0.85 & 25.83 & 4.37 & 0.06 & 0.90 & 1.07 & 0.67 & 3.17 & 0.19 & 0.04 & 11.04 & 0.53 & 99.98 \\
\hline E-AH1 & 2476.80 & Sandstone & 53.20 & 0.74 & 27.13 & 5.85 & 0.03 & 0.98 & 1.03 & 0.66 & 3.96 & 0.13 & 0.03 & 5.58 & 0.63 & 99.95 \\
\hline E-AH1 & 2482.04 & Sandstone & 70.98 & 0.36 & 3.62 & 3.31 & 0.07 & 0.39 & 1.27 & 0.64 & 1.58 & 0.11 & 0.03 & 16.94 & 0.72 & 100.01 \\
\hline E-AH1 & 2483.90 & Sandstone & 60.24 & 0.34 & 3.80 & 1.34 & 0.06 & 0.39 & 1.13 & 0.11 & 1.53 & 0.09 & 0.04 & 30.35 & 0.63 & 100.05 \\
\hline E-AJ1 & 2701.30 & Sandstone & 62.55 & 0.28 & 4.75 & 1.36 & 0.09 & 0.47 & 1.33 & 0.11 & 1.50 & 0.05 & 0.02 & 26.78 & 0.71 & 100.00 \\
\hline E-AJ1 & 2702.50 & Sandstone & 81.58 & 0.26 & 5.83 & 1.32 & 0.08 & 0.45 & 1.33 & 0.42 & 1.57 & 0.07 & 0.02 & 6.62 & 0.52 & 100.07 \\
\hline E-AJ1 & 2703.00 & Sandstone & 61.48 & 0.27 & 3.88 & 1.68 & 0.01 & 0.50 & 1.64 & 0.68 & 1.84 & 0.13 & 0.02 & 27.10 & 0.79 & 100.02 \\
\hline E-AJ1 & 2704.00 & Sandstone & 76.68 & 0.49 & 5.33 & 3.34 & 0.02 & 1.30 & 3.68 & 0.41 & 3.90 & 0.14 & 0.06 & 3.87 & 0.86 & 100.07 \\
\hline E-AJ1 & 2705.00 & Sandstone & 80.49 & 0.40 & 8.28 & 3.42 & 0.07 & 0.92 & 1.17 & 0.81 & 1,46 & 0.17 & 0.02 & 2.28 & 0.55 & 100.03 \\
\hline E-AJ1 & 2721.00 & Sandstone & 71.26 & 0.74 & 6.74 & 2.28 & 0.08 & 0.97 & 1.12 & 0.96 & 2.72 & 0.13 & 0.02 & 12.32 & 0.67 & 100.00 \\
\hline E-AJ1 & 2722.00 & Sandstone & 69.84 & 0.70 & 4.20 & 2.30 & 0.09 & 0.97 & 1.14 & 0.94 & 2.09 & 0.13 & 0.01 & 16.76 & 0.82 & 100.00 \\
\hline E-AJ1 & 2724.00 & Sandstone & 66.66 & 0.67 & 4.01 & 4.74 & 0.05 & 0.90 & 1.09 & 0.89 & 3.61 & 0,13 & 0.03 & 16.50 & 0.73 & 100.00 \\
\hline E-AJ1 & 2726.00 & Sandstone & 74.92 & 0.20 & 7.04 & 3.08 & 0.07 & 0.99 & 0.81 & 0.68 & 1.03 & 0.11 & 0.01 & 10.30 & 0.74 & 99.98 \\
\hline E-AJ1 & 2728.00 & Sandstone & 69.03 & 0.72 & 3.03 & 3.19 & 0.01 & 1.34 & 1.14 & 0.59 & 5.11 & 0.15 & 0.09 & 14.72 & 0.87 & 99.98 \\
\hline E-AJ1 & 2730.00 & Sandstone & 66.01 & 0.57 & 5.00 & 3.99 & 0.02 & 1.37 & 1.42 & 0.23 & 1.59 & 0.19 & 0.05 & 18.71 & 0.98 & 100.13 \\
\hline E-BA1 & 2828.05 & Sandstone & 66.10 & 0.25 & 2.15 & 1.07 & 0.02 & 1.22 & 1.94 & 0,27 & 0.54 & 0.04 & 0.03 & 25.74 & 0.63 & 100.01 \\
\hline E-BA1 & 2832.92 & Sandstone & 74.45 & 0.29 & 6.61 & 1.83 & 0.09 & 1.60 & 2.27 & 0.26 & 0.83 & 0.06 & 0.02 & 10.97 & 0.73 & 100.01 \\
\hline E-BA1 & 2836.01 & Sandstone & 85.10 & 0.16 & 6.09 & 1.23 & 0.04 & 0.74 & 1.98 & 0.30 & 0.54 & 0.11 & 0.02 & 3.52 & 0.62 & 100.44 \\
\hline E-BA1 & 2842.10 & Sandstone & 68.17 & 0.24 & 5.73 & 0.61 & 0.08 & 6.18 & 1.53 & 0.22 & 0.78 & 0.11 & 0.04 & 15.74 & 0.60 & 100.02 \\
\hline E-BB1 & 2538.05 & Sandstone & 67.97 & 0.74 & 3.57 & 3.01 & 0.08 & 1.85 & 1.63 & 0.79 & 3.13 & 0.13 & 0.02 & 16.51 & 0.64 & 100.07 \\
\hline E-BB1 & 2546.00 & Sandstone & 68.00 & 0.29 & 3.79 & 0.27 & 0.01 & 0.42 & 6.31 & 0.34 & 0.72 & 0.11 & 0.03 & 18.84 & 0.86 & 99.98 \\
\hline E-BB1 & 2554.40 & Sandstone & 61.09 & 0.33 & 3.33 & 2.68 & 0.01 & 2.30 & 1.50 & 1.40 & 4.45 & 0.10 & 0.02 & 22.21 & 0.59 & 100.01 \\
\hline E-BB1 & 2659.05 & Sandstone & 62.21 & 0.85 & 4.42 & 4.36 & 0.09 & 2.31 & 1.52 & 0.96 & 3.34 & 0.07 & 0.03 & 18.86 & 0.93 & 99.95 \\
\hline
\end{tabular}


Table 4. Cont.

\begin{tabular}{|c|c|c|c|c|c|c|c|c|c|c|c|c|c|c|c|c|}
\hline Borehole & Depth (m) & Lithology & $\begin{array}{c}\mathrm{SiO}_{2} \\
(\%)\end{array}$ & $\begin{array}{c}\mathrm{TiO}_{2} \\
(\%)\end{array}$ & $\begin{array}{c}\mathrm{Al}_{2} \mathrm{O}_{3} \\
(\%)\end{array}$ & $\begin{array}{c}\mathrm{Fe}_{2} \mathrm{O}_{3} \\
(\%)\end{array}$ & $\begin{array}{c}\mathrm{MnO} \\
(\%)\end{array}$ & $\begin{array}{c}\mathrm{MgO} \\
(\%)\end{array}$ & $\begin{array}{c}\mathrm{CaO} \\
(\%)\end{array}$ & $\begin{array}{c}\mathrm{Na}_{2} \mathrm{O} \\
(\%)\end{array}$ & $\begin{array}{c}\mathrm{K}_{2} \mathrm{O} \\
(\%)\end{array}$ & $\begin{array}{c}\mathrm{P}_{2} \mathrm{O}_{5} \\
(\%)\end{array}$ & $\begin{array}{c}\mathrm{Cr}_{2} \mathrm{O}_{3} \\
(\%)\end{array}$ & $\begin{array}{l}\text { LOI } \\
(\%)\end{array}$ & $\begin{array}{c}\mathrm{H}_{2} \mathrm{O}^{-} \\
(\%)\end{array}$ & $\begin{array}{c}\text { Total } \\
(\%)\end{array}$ \\
\hline E-BB1 & 2663.18 & Sandstone & 64.15 & 0.79 & 2.82 & 2.73 & 0.09 & 2.14 & 0.93 & 1.06 & 2.91 & 0.09 & 0.01 & 21.39 & 0.87 & 99.98 \\
\hline E-BB1 & 2720.55 & Sandstone & 62.09 & 0.67 & 5.87 & 2.35 & 0.09 & 1.77 & 1.58 & 0.71 & 2.43 & 0.08 & 0.04 & 21.60 & 0.71 & 99.99 \\
\hline E-BB1 & 2722.55 & Sandstone & 63.65 & 0.10 & 2.62 & 1.01 & 0.01 & 1.28 & 1.09 & 0.77 & 3.44 & 0.15 & 0.05 & 25.01 & 0.78 & 99.96 \\
\hline E-BB1 & 2849.05 & Sandstone & 68.54 & 0.24 & 4.23 & 3.32 & 0.05 & 1.07 & 1.08 & 0.45 & 4.89 & 0.14 & 0.01 & 15.36 & 0.68 & 100.05 \\
\hline E-BB1 & 2852.03 & Sandstone & 63.29 & 0.52 & 3.61 & 0.44 & 0.03 & 1.02 & 1.17 & 0.65 & 1.74 & 0.07 & 0.01 & 26.73 & 0.75 & 100.03 \\
\hline E-BB1 & 2856.05 & Sandstone & 65.72 & 0.45 & 6.50 & 0.96 & 0.05 & 5.68 & 1.10 & 0.52 & 1.04 & 0.09 & 0.01 & 17.26 & 0.62 & 99.99 \\
\hline E-BB1 & 2872.05 & Sandstone & 75.24 & 0.52 & 3.83 & 1.07 & 0.05 & 1.08 & 1.34 & 1.19 & 1.59 & 0.14 & 0.01 & 13.48 & 0.51 & 100.04 \\
\hline E-BB1 & 2894.05 & Sandstone & 52.21 & 0.67 & 24.08 & 4.49 & 0.03 & 1.84 & 2.39 & 0.89 & 3.32 & 0.08 & 0.04 & 9.54 & 0.54 & 100.10 \\
\hline E-BB1 & 3280.06 & Sandstone & 52.28 & 0.74 & 22.53 & 4.86 & 0.02 & 1.80 & 1.96 & 0.96 & 3.96 & 0.18 & 0.01 & 9.90 & 0.84 & 100.02 \\
\hline E-BB1 & 3284.87 & Sandstone & 58.02 & 0.57 & 23.87 & 3.89 & 0.04 & 2.16 & 4.82 & 0.97 & 2.82 & 0.13 & 0.03 & 1.81 & 0.90 & 100.02 \\
\hline E-BB1 & 3290.31 & Sandstone & 52.68 & 0.66 & 23.01 & 3.49 & 0.01 & 1.71 & 1.46 & 1.03 & 3.04 & 0.09 & 0.01 & 12.11 & 0.68 & 99.96 \\
\hline E-D3 & 3268.58 & Sandstone & 54.13 & 0.61 & 23.99 & 3.04 & 0.01 & 1.56 & 3.39 & 0.87 & 2.56 & 0.06 & 0.02 & 9.17 & 0.61 & 100.01 \\
\hline E-D3 & 3527.50 & Sandstone & 69.25 & 0.53 & 4.17 & 1.09 & 0.02 & 0.79 & 7.68 & 0.56 & 1.05 & 0.15 & 0.01 & 14.00 & 0.78 & 100.05 \\
\hline E-D3 & 3733.25 & Sandstone & 76.58 & 0.53 & 3.60 & 1.14 & 0.01 & 1.28 & 1.23 & 1.03 & 1.64 & 0.05 & 0.01 & 12.24 & 0.73 & 100.05 \\
\hline E-D3 & 3736.03 & Sandstone & 56.20 & 0.50 & 24.02 & 3.34 & 0.09 & 1.35 & 1.44 & 0.89 & 2.03 & 0.14 & 0.02 & 9.28 & 0.77 & 100.04 \\
\hline E-D3 & 3737.00 & Sandstone & 79.27 & 0.20 & 4.61 & 1.48 & 0.01 & 1.09 & 3.11 & 0.94 & 0.86 & 0.12 & 0.06 & 7.53 & 0.61 & 99.90 \\
\hline
\end{tabular}

NB. LOI-Loss of ignition. 
Table 5. Modal compositions of the studied sandstones from the Bredasdorp Basin.

\begin{tabular}{|c|c|c|c|c|c|c|c|c|c|c|c|c|c|c|c|c|}
\hline \multirow{2}{*}{ Borehole } & \multirow{2}{*}{ Depth (m) } & \multirow{2}{*}{ Qt (\%) } & \multirow{2}{*}{ Qm (\%) } & \multirow{2}{*}{ Qp (\%) } & \multirow{2}{*}{ F (\%) } & \multirow{2}{*}{ L (\%) } & \multirow{2}{*}{ GL (\%) } & \multirow{2}{*}{ Acc (\%) } & \multirow{2}{*}{ Cmt (\%) } & \multirow{2}{*}{ Mx (\%) } & \multicolumn{3}{|c|}{ QmFLt (\%) } & \multicolumn{3}{|c|}{ QtFL (\%) } \\
\hline & & & & & & & & & & & $\mathrm{Qm}$ & $\mathbf{F}$ & $\mathbf{L t}$ & $Q t$ & $\mathbf{F}$ & L \\
\hline \multirow{5}{*}{ E-AH1 } & 2471.0 & 59.0 & 57.6 & 1.4 & 13.4 & 7.4 & 4.2 & 0.2 & 3.8 & 12.0 & 72.2 & 16.8 & 11.0 & 73.9 & 16.8 & 9.3 \\
\hline & 2473.0 & 52.2 & 52.0 & 0.2 & 13.0 & 9.2 & 4.4 & 1.0 & 6.4 & 13.8 & 69.9 & 17.5 & 12.6 & 70.2 & 17.5 & 12.4 \\
\hline & 2475.0 & 58.8 & 56.0 & 2.8 & 14.2 & 5.8 & 5.0 & 0.3 & 6.4 & 9.5 & 71.1 & 18.0 & 10.9 & 74.6 & 18.0 & 7.4 \\
\hline & 2482.0 & 65.2 & 62.2 & 3.0 & 10.0 & 6.4 & 3.0 & 0.1 & 5.0 & 10.3 & 76.2 & 12.3 & 11.5 & 79.9 & 12.3 & 7.8 \\
\hline & 2483.0 & 65.8 & 63.8 & 2.0 & 14.2 & 5.2 & 5.0 & 1.0 & 5.6 & 3.2 & 74.9 & 16.7 & 8.5 & 77.2 & 16.7 & 6.1 \\
\hline \multirow{11}{*}{ E-AJ1 } & 2701.3 & 66.2 & 64.2 & 2.0 & 13.8 & 6.6 & 4.2 & 0.3 & 4.2 & 4.7 & 74.1 & 15.9 & 9.9 & 76.4 & 15.9 & 7.6 \\
\hline & 2702.5 & 68.4 & 66.6 & 1.8 & 14.2 & 5.6 & 1.0 & 0.1 & 6.4 & 4.3 & 75.5 & 16.1 & 8.4 & 77.6 & 16.1 & 6.3 \\
\hline & 2703.0 & 61.0 & 59.0 & 2.0 & 14.0 & 5.0 & 4.0 & 0.8 & 6.4 & 8.8 & 73.8 & 17.5 & 8.8 & 76.3 & 17.5 & 6.3 \\
\hline & 2704.0 & 52.2 & 51.8 & 0.4 & 15.2 & 6.2 & 4.0 & 0.4 & 8.6 & 13.4 & 70.4 & 20.7 & 9.0 & 70.9 & 20.7 & 8.4 \\
\hline & 2705.0 & 59.8 & 59.6 & 0.2 & 18.8 & 6.2 & 3.0 & 0.4 & 4.2 & 7.6 & 70.3 & 22.2 & 7.5 & 70.5 & 22.2 & 7.3 \\
\hline & 2721.0 & 60.4 & 60.2 & 0.2 & 19.8 & 5.8 & 3.0 & 0.2 & 3.0 & 7.8 & 70.0 & 23.0 & 7.0 & 70.2 & 23.0 & 6.7 \\
\hline & 2722.0 & 66.0 & 65.4 & 0.6 & 15.2 & 7.8 & 2.2 & 0.2 & 1.8 & 6.8 & 73.5 & 17.1 & 9.4 & 74.2 & 17.1 & 8.8 \\
\hline & 2724.0 & 60.2 & 59.8 & 0.4 & 10.6 & 5.6 & 3.6 & 0.8 & 9.4 & 9.8 & 78.3 & 13.9 & 7.9 & 78.8 & 13.9 & 7.3 \\
\hline & 2726.0 & 60.6 & 59.2 & 1.4 & 13.6 & 7.2 & 2.4 & 0.5 & 5.8 & 9.9 & 72.7 & 16.7 & 10.6 & 74.4 & 16.7 & 8.8 \\
\hline & 2728.0 & 62.0 & 61.0 & 1.0 & 17.6 & 5.8 & 3.2 & 0.6 & 3.8 & 7.0 & 71.4 & 20.6 & 8.0 & 72.6 & 20.6 & 6.8 \\
\hline & 2730.0 & 61.0 & 60.4 & 0.6 & 15.2 & 9.6 & 1.4 & 0.1 & 5.8 & 6.9 & 70.4 & 17.7 & 11.9 & 71.1 & 17.7 & 11.2 \\
\hline \multirow{4}{*}{ E-BA1 } & 2828.0 & 60.0 & 59.4 & 0.6 & 13.8 & 8.6 & 3.6 & 0.0 & 3.6 & 10.4 & 72.1 & 16.7 & 11.2 & 72.8 & 16.7 & 10.4 \\
\hline & 2832.0 & 68.4 & 67.6 & 0.8 & 13.8 & 8.0 & 2.0 & 0.2 & 2.4 & 5.2 & 74.9 & 15.3 & 9.8 & 75.8 & 15.3 & 8.9 \\
\hline & 2836.0 & 63.2 & 62.8 & 0.4 & 14.6 & 7.2 & 3.6 & 0.6 & 3.6 & 7.2 & 73.9 & 17.2 & 8.9 & 74.4 & 17.2 & 8.5 \\
\hline & 2842.0 & 59.6 & 59.4 & 0.2 & 13.8 & 7.0 & 3.4 & 0.2 & 6.0 & 10.0 & 73.9 & 17.2 & 9.0 & 74.1 & 17.2 & 8.7 \\
\hline \multirow{10}{*}{ E-BB1 } & 2537.7 & 62.0 & 61.8 & 0.2 & 14.0 & 6.0 & 2.0 & 0.2 & 6.2 & 9.6 & 75.4 & 17.1 & 7.6 & 75.6 & 17.1 & 7.3 \\
\hline & 2546.0 & 65.0 & 64.4 & 0.6 & 15.8 & 6.8 & 2.0 & 0.0 & 2.4 & 8.0 & 73.5 & 18.0 & 8.4 & 74.2 & 18.0 & 7.8 \\
\hline & 2553.0 & 57.8 & 57.4 & 0.4 & 13.6 & 6.0 & 3.6 & 0.3 & 7.0 & 11.7 & 74.2 & 17.6 & 8.3 & 74.7 & 17.6 & 7.8 \\
\hline & 2659.0 & 62.0 & 60.6 & 1.4 & 15.6 & 6.0 & 2.4 & 0.8 & 3.6 & 9.6 & 72.5 & 18.7 & 8.9 & 74.2 & 18.7 & 7.2 \\
\hline & 2663.0 & 61.6 & 60.8 & 0.8 & 16.4 & 7.8 & 2.4 & 0.6 & 3.2 & 8.0 & 70.9 & 19.1 & 10.0 & 71.8 & 19.1 & 9.1 \\
\hline & 2721.0 & 60.2 & 60.0 & 0.2 & 15.0 & 6.6 & 3.0 & 0.6 & 4.6 & 10.0 & 73.3 & 18.3 & 8.3 & 73.6 & 18.3 & 8.1 \\
\hline & 2723.0 & 57.6 & 56.6 & 1.0 & 14.8 & 6.8 & 4.2 & 0.0 & 5.6 & 11.0 & 71.5 & 18.7 & 9.8 & 72.7 & 18.7 & 8.6 \\
\hline & 2849.0 & 61.4 & 60.4 & 1.0 & 13.8 & 7.6 & 2.4 & 0.2 & 5.0 & 9.6 & 72.9 & 16.7 & 10.4 & 74.2 & 16.7 & 9.2 \\
\hline & 2853.0 & 60.0 & 59.0 & 1.0 & 11.0 & 5.2 & 3.0 & 0.8 & 8.0 & 12.0 & 77.4 & 14.4 & 8.1 & 78.7 & 14.4 & 6.8 \\
\hline & 2855.0 & 61.0 & 60.6 & 0.4 & 14.0 & 5.8 & 1.4 & 0.2 & 5.8 & 11.8 & 75.0 & 17.3 & 7.7 & 75.5 & 17.3 & 7.2 \\
\hline
\end{tabular}


Table 5. Cont.

\begin{tabular}{|c|c|c|c|c|c|c|c|c|c|c|c|c|c|c|c|c|}
\hline \multirow{2}{*}{ Borehole } & \multirow{2}{*}{ Depth (m) } & \multirow{2}{*}{ Qt (\%) } & \multirow{2}{*}{ Qm (\%) } & \multirow{2}{*}{ Qp (\%) } & \multirow{2}{*}{$F(\%)$} & \multirow{2}{*}{ L (\%) } & \multirow{2}{*}{ GL (\%) } & \multirow{2}{*}{$\operatorname{Acc}(\%)$} & \multirow{2}{*}{ Cmt (\%) } & \multirow{2}{*}{ Mx (\%) } & \multicolumn{3}{|c|}{ QmFLt (\%) } & \multicolumn{3}{|c|}{ QtFL (\%) } \\
\hline & & & & & & & & & & & Qm & $\mathbf{F}$ & Lt & Qt & $\mathbf{F}$ & L \\
\hline & 2872.0 & 57.2 & 57.0 & 0.2 & 15.2 & 7.0 & 2.2 & 0.1 & 6.0 & 12.3 & 71.8 & 19.1 & 9.1 & 72.0 & 19.1 & 8.8 \\
\hline & 2873.8 & 56.0 & 55.6 & 0.4 & 15.6 & 7.8 & 4.0 & 0.4 & 6.6 & 9.6 & 70.0 & 19.6 & 10.3 & 70.5 & 19.6 & 9.8 \\
\hline & 3280.4 & 59.8 & 59.6 & 0.2 & 17.6 & 8.0 & 1.8 & 0.6 & 4.2 & 8.0 & 69.8 & 20.6 & 9.6 & 70.0 & 20.6 & 9.4 \\
\hline & $3284 . .0$ & 57.6 & 57.4 & 0.2 & 12.4 & 7.2 & 2.0 & 0.2 & 9.0 & 11.6 & 74.4 & 16.1 & 9.6 & 74.6 & 16.1 & 9.3 \\
\hline & 3292.7 & 59.8 & 59.4 & 0.4 & 16.2 & 7.2 & 2.6 & 0.0 & 5.6 & 8.6 & 71.4 & 19.5 & 9.1 & 71.9 & 19.5 & 8.7 \\
\hline \multirow{5}{*}{ E-D3 } & 3268.0 & 63.0 & 62.2 & 0.8 & 15.0 & 6.4 & 2.4 & 0.8 & 4.4 & 8.0 & 73.7 & 17.8 & 8.5 & 74.6 & 17.8 & 7.6 \\
\hline & 3269.0 & 58.4 & 58.0 & 0.4 & 14.4 & 6.8 & 3.6 & 0.0 & 5.0 & 11.8 & 72.9 & 18.1 & 9.0 & 73.4 & 18.1 & 8.5 \\
\hline & 3526.0 & 62.0 & 60.8 & 1.2 & 14.0 & 6.2 & 3.2 & 0.2 & 5.4 & 9.0 & 74.0 & 17.0 & 9.0 & 75.4 & 17.0 & 7.5 \\
\hline & 3528.5 & 59.2 & 58.2 & 1.0 & 13.4 & 5.2 & 2.2 & 0.0 & 6.2 & 13.8 & 74.8 & 17.2 & 8.0 & 76.1 & 17.2 & 6.7 \\
\hline & 3529.5 & 62.8 & 62.2 & 0.6 & 15.6 & 6.2 & 2.6 & 0.1 & 4.2 & 8.5 & 73.5 & 18.4 & 8.0 & 74.2 & 18.4 & 7.3 \\
\hline
\end{tabular}

Note: $\mathrm{Qt}=$ total quartz $(\mathrm{Qm}+\mathrm{Qp}), \mathrm{Qm}=$ monocrystalline quartz, $\mathrm{Qp}=$ Polycrystalline quartz, $\mathrm{F}=$ Feldspar, $\mathrm{L}=$ Unstable siliciclastic lithic fragments $(\mathrm{Lv}+\mathrm{Ls}+\mathrm{Lm}), \mathrm{Ls}=\mathrm{Sedimentary}$ lithic fragment, $\mathrm{Lv}=$ Volcanic plus igneous lithic fragment, $\mathrm{Lm}=$ Metamorphic lithic fragment, $\mathrm{Lt}=$ Total siliciclastic lithic fragment $(\mathrm{L}+\mathrm{Qp}), \mathrm{GL}=\mathrm{Glauconite}, \mathrm{Acc}=\mathrm{Accessory}$ minerals, $\mathrm{cmt}=$ Cement, $\mathrm{Mx}=$ Matrix 
Table 6. Mineralogical composition of the studied sandstone samples from XRD.

\begin{tabular}{|c|c|c|c|c|c|c|c|c|c|c|c|c|c|c|}
\hline Borehole & $\begin{array}{c}\text { Depth } \\
\text { (m) }\end{array}$ & $\begin{array}{c}\text { Quartz } \\
(\%)\end{array}$ & $\begin{array}{c}\text { Pyrite } \\
(\%)\end{array}$ & $\begin{array}{c}\text { Muscovite } \\
(\%)\end{array}$ & $\begin{array}{c}\text { Kaolinite } \\
(\%)\end{array}$ & $\begin{array}{c}\text { Plagioclase } \\
(\%)\end{array}$ & $\begin{array}{c}\text { Glauconite } \\
(\%)\end{array}$ & $\begin{array}{c}\text { Rutile } \\
(\%)\end{array}$ & $\begin{array}{c}\text { Dolomite } \\
\text { (\%) }\end{array}$ & $\begin{array}{c}\text { Calcite/ } \\
(\%)\end{array}$ & $\begin{array}{c}\text { Chlorite } \\
(\%)\end{array}$ & $\begin{array}{c}\text { Microcline } \\
\text { (\%) }\end{array}$ & $\begin{array}{c}\text { Smectite } \\
(\%)\end{array}$ & $\begin{array}{c}\text { Illite } \\
(\%)\end{array}$ \\
\hline \multirow{5}{*}{ E-AH1 } & 2471 & 56.1 & 2.1 & 5.8 & 13.6 & 6.1 & 4.1 & 0.0 & 0.4 & 9.4 & 0.1 & 1.3 & 0.6 & 0.5 \\
\hline & 2475 & 67.6 & 0.9 & 1.2 & 4.1 & 7.3 & 1.7 & 0.0 & 0.3 & 12.8 & 0.6 & 1.6 & 0.0 & 1.6 \\
\hline & 2478 & 69.4 & 1.0 & 0.9 & 7.4 & 8.9 & 0.6 & 0.1 & 0.1 & 6.4 & 0.3 & 4.5 & 0.1 & 0.0 \\
\hline & 2481 & 68.2 & 0.4 & 3.5 & 3.9 & 9.2 & 1.8 & 0.2 & 0.3 & 7.9 & 0.6 & 3.7 & 0.1 & 0.0 \\
\hline & 2483 & 67.4 & 0.3 & 2.2 & 5.3 & 9.7 & 2.0 & 0.0 & 0.2 & 9.4 & 0.4 & 2.1 & 0.3 & 0.5 \\
\hline \multirow{7}{*}{ E-AJ1 } & 2703 & 73.7 & 0.2 & 1.1 & 4.0 & 8.3 & 0.1 & 0.1 & 0.6 & 9.0 & 0.3 & 2.1 & 0.1 & 0.3 \\
\hline & 2721 & 68.5 & 0.2 & 1.2 & 5.0 & 9.2 & 1.9 & 0.1 & 0.7 & 10.4 & 0.8 & 1.7 & 0.0 & 0.2 \\
\hline & 2726 & 69.4 & 0.3 & 1.0 & 1.6 & 10.2 & 0.6 & 0.5 & 2.4 & 10.9 & 1.3 & 1.3 & 0.0 & 0.4 \\
\hline & 2730 & 63.5 & 0.1 & 5.6 & 10.4 & 11.1 & 0.2 & 0.4 & 0.2 & 7.1 & 0.2 & 1.1 & 0.1 & 0.0 \\
\hline & 2970 & 71.5 & 0.2 & 5.9 & 3.7 & 12.8 & 1.3 & 0.1 & 0.0 & 3.0 & 0.3 & 0.8 & 0.2 & 0.3 \\
\hline & 2982 & 69.7 & 0.6 & 4.7 & 6.6 & 14.5 & 0.6 & 0.3 & 0.2 & 1.8 & 0.1 & 0.4 & 0.2 & 0.2 \\
\hline & 3039 & 68.4 & 0.4 & 3.2 & 5.1 & 12.1 & 0.9 & 0.6 & 0.3 & 5.4 & 1.0 & 2.1 & 0.1 & 0.3 \\
\hline \multirow{5}{*}{ E-BA1 } & 2828 & 63.7 & 5.9 & 1.5 & 4.2 & 10.1 & 0.3 & 0.2 & 1.8 & 7.1 & 2.7 & 1.6 & 0.1 & 0.8 \\
\hline & 2832 & 68.7 & 0.3 & 2.2 & 3.7 & 14.5 & 0.2 & 0.1 & 0.1 & 8.1 & 0.5 & 1.4 & 0.0 & 0.0 \\
\hline & 2838 & 69.4 & 0.2 & 1.7 & 7.1 & 8.5 & 0.4 & 0.2 & 0.1 & 10.2 & 0.1 & 1.6 & 0.2 & 0.0 \\
\hline & 2843 & 61.9 & 0.7 & 2.0 & 6.8 & 9.3 & 0.3 & 0.3 & 0.0 & 16.8 & 0.3 & 1.3 & 0.2 & 0.1 \\
\hline & 2850 & 61.7 & 0.7 & 1.8 & 5.3 & 13.4 & 1.7 & 0.1 & 0.2 & 12.9 & 0.3 & 1.0 & 0.1 & 0.5 \\
\hline \multirow{9}{*}{ E-BB1 } & 2539 & 60.7 & 4.4 & 13.3 & 6.2 & 10.6 & 0.6 & 0.3 & 0.0 & 0.4 & 2.8 & 0.7 & 0.0 & 0.0 \\
\hline & 2548 & 60.1 & 2.5 & 2.6 & 6.9 & 9.8 & 0.1 & 0.1 & 0.0 & 13.5 & 1.2 & 2.8 & 0.1 & 0.1 \\
\hline & 2556 & 71.4 & 1.7 & 4.1 & 4.6 & 10.3 & 0.7 & 0.1 & 0.1 & 5.0 & 0.9 & 0.8 & 0.0 & 0.0 \\
\hline & 2662 & 70.4 & 1.9 & 4.2 & 4.7 & 9.6 & 0.3 & 0.19 & 0.2 & 4.2 & 1.0 & 3.2 & 0.0 & 0.0 \\
\hline & 2721 & 63.9 & 0.9 & 2.8 & 6.2 & 14.7 & 0.2 & 0.2 & 0.0 & 7.7 & 1.4 & 1.2 & 0.1 & 0.5 \\
\hline & 2849 & 67.6 & 0.2 & 2.1 & 4.9 & 15.2 & 0.1 & 0.0 & 0.1 & 6.9 & 0.8 & 1.4 & 0.0 & 0.7 \\
\hline & 2863 & 65.2 & 0.8 & 2.0 & 7.8 & 13.0 & 0.7 & 0.0 & 0.1 & 8.0 & 0.7 & 1.0 & 0.1 & 0.4 \\
\hline & 3284 & 70.0 & 0.3 & 1.4 & 5.2 & 8.9 & 0.4 & 0.0 & 0.2 & 11.7 & 0.4 & 1.0 & 0.0 & 0.2 \\
\hline & 3294 & 67.4 & 0.5 & 3.0 & 6.0 & 11.8 & 0.5 & 0.6 & 0.5 & 8.2 & 0.8 & 0.5 & 0.0 & 0.0 \\
\hline \multirow{4}{*}{ E-D3 } & 3261 & 68.4 & 1.0 & 2.5 & 5.1 & 10.6 & 0.4 & 0.5 & 0.6 & 7.1 & 0.7 & 2.6 & 0.1 & 0.2 \\
\hline & 3269 & 65.3 & 0.20 & 6.6 & 7.1 & 9.5 & 0.5 & 0.5 & 0.3 & 5.3 & 2.1 & 1.0 & 0.0 & 0.2 \\
\hline & 3526 & 67.5 & 0.3 & 3.4 & 6.0 & 12.3 & 1.1 & 0.3 & 0.2 & 4.3 & 1.6 & 2.4 & 0.0 & 0.4 \\
\hline & 3529 & 70.8 & 0.2 & 2.8 & 5.4 & 11.0 & 1.3 & 0.2 & 0.1 & 6.2 & 0.4 & 0.9 & 0.3 & 0.4 \\
\hline
\end{tabular}




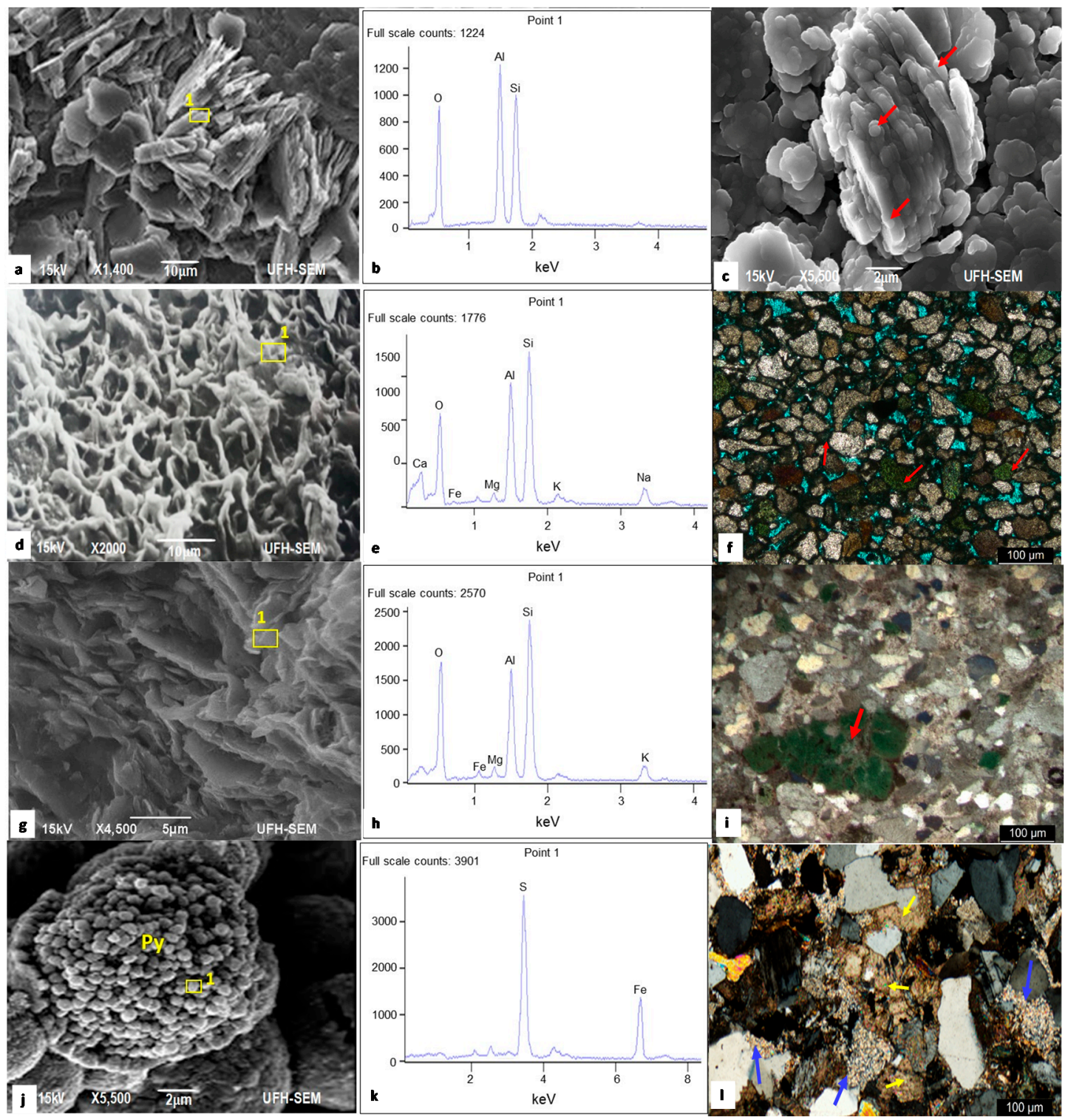

Figure 4. (a) SEM image showing aggregates of booklet-like kaolinite (yellow arrows) in sandstone from Borehole E-AH1 (yellow arrows); (b) EDX spectrum showing elemental compositions of kaolinite at point 1 in Figure 4a; (c) SEM image showing rod-shaped illite growth (red arrows) from the kaolinite in sandstone from Borehole E-AH1; (d) SEM image showing honey-comb shaped smectite in sandstone from Borehole E-BB1; (e) EDX spectrum showing elemental compositions of smectite at point 1 in Figure 4d; (f) Photomicrograph of sandstone from borehole E-AJ1 showing precipitation of glauconite in intergranular pore spaces (red arrows); (g) SEM image showing authigenic illite with incipient lath-like or fibrous forms in sandstone from Borehole E-BA1; (h) EDX spectrum showing elemental compositions of illite at point 1 in Figure 4g. (i) Partial replacement of feldspar and matrix by glauconite (red arrow); (j) SEM image of authigenic framboid pyrite in sandstone from Borehole E-BB1; (k) EDX spectrum showing elemental composition of pyrite at point 1 in Figure 4j; (l) Photomicrograph showing partial replacement of feldspar by clay minerals (red arrows) and calcite (yellow arrows). 


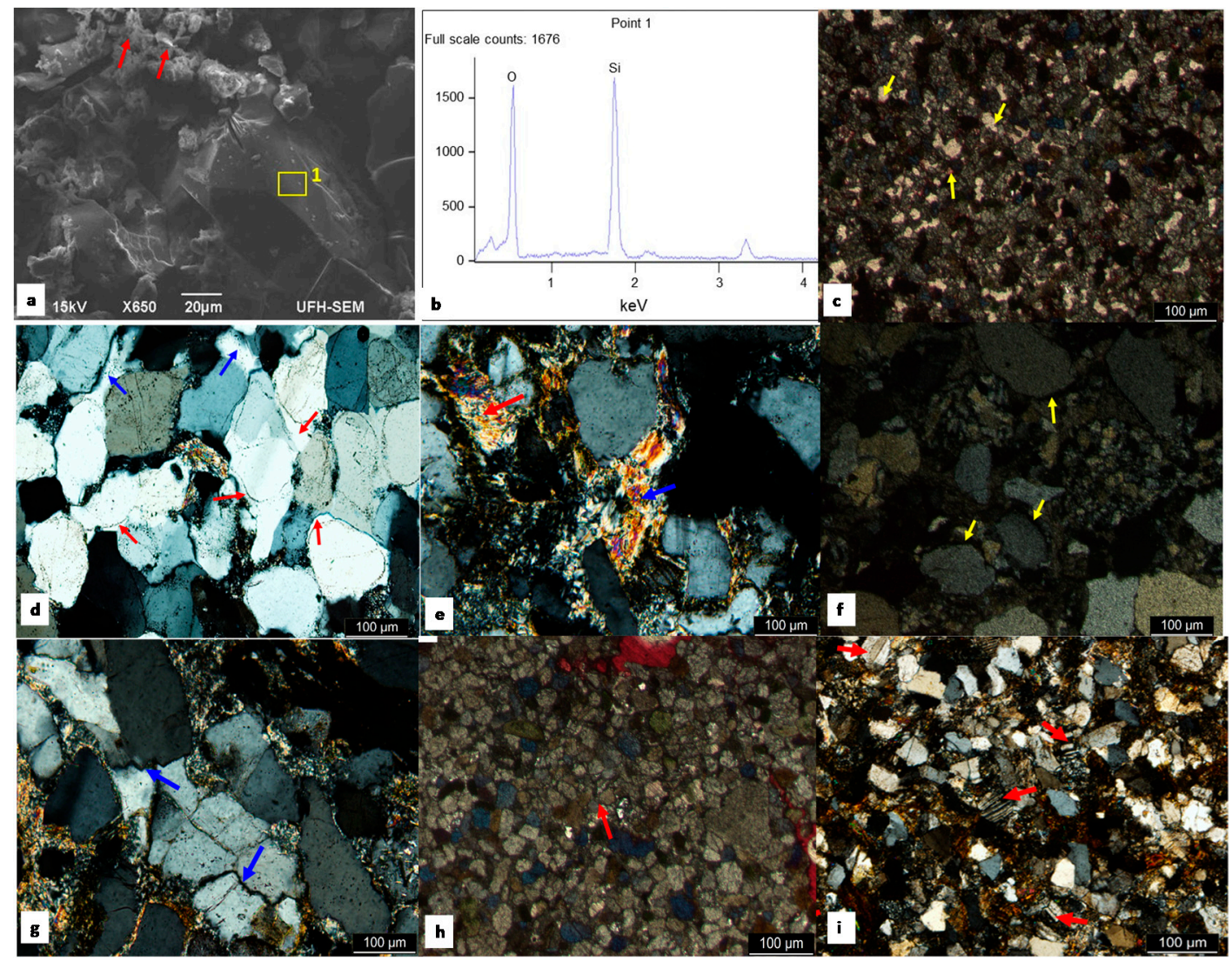

Figure 5. (a) SEM image of pore-filling rosette-like chlorite (red arrow) and euhedral quartz in sandstone from Borehole E-D3; (b) EDX spectrum showing elemental composition of quartz at point 1 in Figure $4 \mathrm{~m}$; (c) Photomicrograph of sandstone from borehole E-BB1 showing ferroan calcite (yellow arrows) filling intergranular pores; (d) Photomicrograph of sandstone from Borehole E-D3 showing authigenic quartz cement (blue arrows) and quartz overgrowths (red arrows); (e) Photomicrograph of sandstone from borehole E-BB1 showing Recrystallization of kaolinite and smectite to illite (red arrow) and muscovite (blue arrow); (f) Photomicrograph showing authigenic pyrite cement in the form of thin rim or patch surrounding framework grains (yellow arrows); (g) Photomicrograph of sandstone from borehole E-BA1 showing Dissolution cracks (blue arrows) formed by pressure dissolution along grain boundaries; (h) Photomicrograph of sandstone from Borehole E-BA1 showing clay matrix with chlorite component (brownish colour) and ferroan calcite (reddish colour); (i) Photomicrograph of sandstone from Borehole E-BA1 showing partial replacement of microcline by albite (red arrows). 


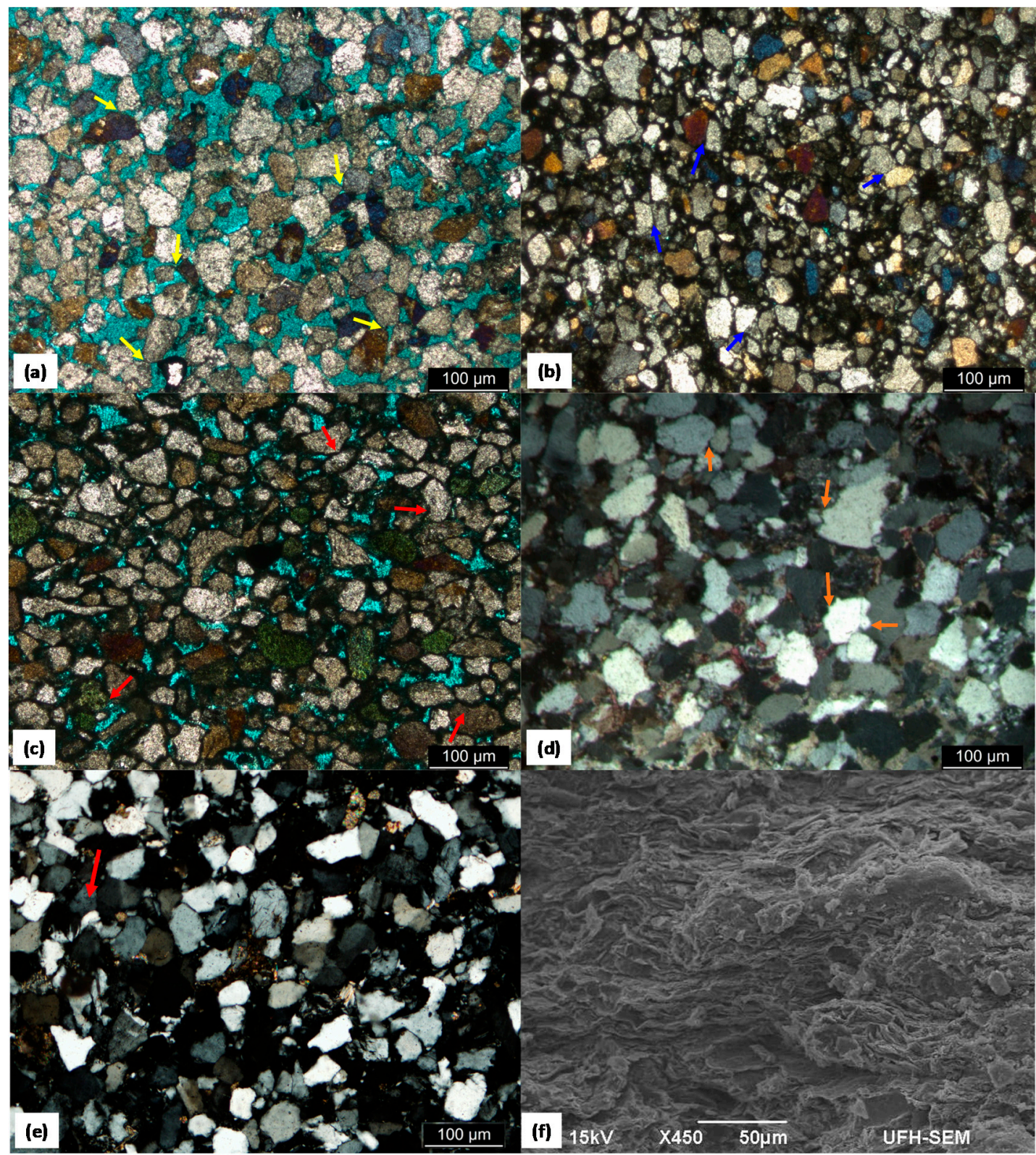

Figure 6. Thin section photomicrograph of sandstone showing different grain to grain contact patterns due to increasing burial depth: (a) Point contact (yellow arrows); (b) Long contacts (blue arrows); (c) Convano-convex contacts (red arrows); (d) Sutured contacts (brown arrows); (e) Grain deformation; (f) Grain alignment and deformation: SEM (BSE) photomicrograph showing orientation and deformation of clays as a result of compaction.

\subsection{Porosity and Permeability}

The observed primary porosities are intergranular (pore space between grains) and microporosity (matrix micro pores), while the secondary porosities are secondary intragranular, dissolution and fractured pores (Table 7; Figure 7). The point count porosity values for the sandstones varies between $1.9 \%$ and $22.7 \%$, averaging $11.8 \%$ (Table 8 ). Specifically, the point count porosity for exploration wells E-AH1, E-AJ1, E-BA1, E-BB1 and E-D3 ranges between 11.4-22.7\%, 9.4-17.2\%, 22.4-13.9\%, 3.7-15.3\%, and $1.4-14.3 \%$, respectively. The summary of the observed reservoir properties of the sandstones is shown in Table 8. 
Table 7. Observed pores in the sandstones of the Bredasdorp Basin.

\begin{tabular}{cccc}
\hline & Type of Pore & Method of Formation & Characteristics \\
\hline Primary pores & Primary intergranular pores & $\begin{array}{c}\text { Compaction and contraction } \\
\text { between grains }\end{array}$ & Point to line contact of grains \\
\cline { 2 - 4 } Secondary pores & $\begin{array}{c}\text { Matrix micro pores } \\
\text { (Micro porosity) }\end{array}$ & $\begin{array}{c}\text { Pores formed by the sustained } \\
\text { function between matrix grains }\end{array}$ & $\begin{array}{c}\text { Pores between authigenic } \\
\text { clay minerals }\end{array}$ \\
\cline { 2 - 4 } & Solution pores & $\begin{array}{c}\text { Formed by dissolution of } \\
\text { cements and framework grains }\end{array}$ & $\begin{array}{c}\text { Corrosion of the edge of grains } \\
\text { and cements }\end{array}$ \\
\cline { 2 - 4 } & Secondary intragranular pores & $\begin{array}{c}\text { Pores produced by the } \\
\text { dissolution of detrital feldspars, } \\
\text { lithic fragments and cement } \\
\text { material (i.e., calcite cement). }\end{array}$ & $\begin{array}{c}\text { Corrosion inside of } \\
\text { feldspar grains }\end{array}$ \\
\cline { 2 - 4 } & $\begin{array}{c}\text { Fracturing of rocks by } \\
\text { differential compaction and } \\
\text { tectonic forces }\end{array}$ & $\begin{array}{c}\text { Presence of cracks or the grains } \\
\text { are folded }\end{array}$ \\
\hline
\end{tabular}

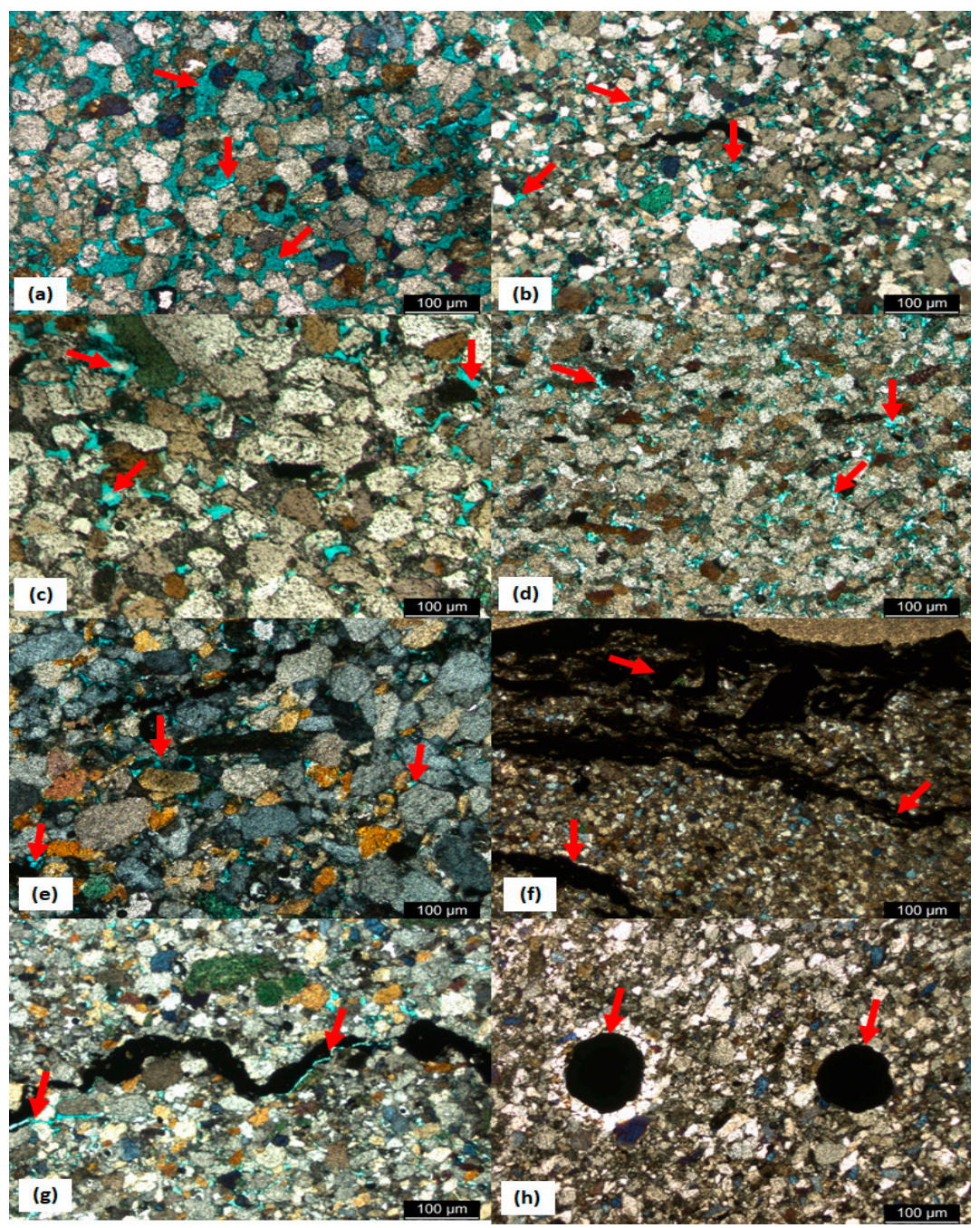

Figure 7. Thin section photomicrograph of sandstone showing some reservoir properties: (a) Primary pore spaces (green areas, red arrows) in Borehole E-AH1 at a depth of $2477 \mathrm{~m}$; (b) pore spaces (green areas, red arrows) in Borehole E-AJ1 at a depth of $2704 \mathrm{~m}$; (c) Pore spaces (green areas, red arrows) in Borehole E-BA1 at a depth of $2835 \mathrm{~m}$; (d) Pore spaces (green areas, red arrows) in Borehole E-BB1 at a depth of $2852 \mathrm{~m}$; (e) Pore spaces (Top: hydrocarbon fills to previously fractured zone, red arrows point to pore space) in Borehole E-D3 at a depth of $3528 \mathrm{~m} ;(\mathbf{f}, \mathbf{g})$ Solid bitumens filling the fractured pores (red arrows) in Borehole E-BB1; (h) Oil emplacement in Borehole E-AJ1. 
Table 8. The summary of the reservoir properties of the sandstones of the Bredasdorp Basin.

\begin{tabular}{|c|c|c|c|c|c|c|}
\hline Borehole & Depth (m) & $\begin{array}{l}\text { Gas Expansion } \\
\text { Porosity (\%) }\end{array}$ & $\begin{array}{l}\text { Point Count } \\
\text { Porosity (\%) }\end{array}$ & $\begin{array}{c}\text { Klinkenberg } \\
\text { Corrected } \\
\text { Permeability (mD) }\end{array}$ & $\begin{array}{c}\text { Dominant Cement } \\
\text { under SEM and } \\
\text { Petrography }\end{array}$ & $\begin{array}{l}\text { Effect of Diagenesis on the } \\
\text { Reservoir Properties }\end{array}$ \\
\hline \multirow{5}{*}{ E-AH1 } & 2471.08 & 16.80 & 14.80 & 30.00 & \multirow{5}{*}{$\begin{array}{l}\text { Quartz, kaolinite, } \\
\text { non-ferroan calcite }\end{array}$} & \multirow{5}{*}{$\begin{array}{l}\text { Porosity and permeability are generally } \\
\text { good. Secondary porosities are well } \\
\text { developed. The abundance of elongate } \\
\text { and oversized pores, and the } \\
\text { uncompacted nature of the sandstones } \\
\text { suggest that much of the porosity is } \\
\text { secondary, after dissolution of an early } \\
\text { intergranular cement and detrital grains }\end{array}$} \\
\hline & 2473.98 & 14.20 & 11.40 & 22.00 & & \\
\hline & 2476.80 & 14.50 & 15.60 & 30.00 & & \\
\hline & 2482.04 & 20.60 & 22.70 & 61.00 & & \\
\hline & 2483.90 & 16.30 & 17.40 & 49.00 & & \\
\hline \multirow{11}{*}{ E-AJ1 } & 2701.30 & 16.80 & 15.20 & 19.40 & \multirow[t]{2}{*}{$\begin{array}{l}\text { Quartz and } \\
\text { ferroan calcite }\end{array}$} & \multirow{2}{*}{$\begin{array}{l}\text { Porosity is good and permeability is } \\
\text { moderate to good. Quartz commonly } \\
\text { fills the secondary pores and the } \\
\text { pseudomatrix in some places results in } \\
\text { the reduced permeability }\end{array}$} \\
\hline & 2702.50 & 12.50 & 11.80 & 4.40 & & \\
\hline & 2703.00 & 11.70 & 12.60 & 2.90 & \multirow{3}{*}{$\begin{array}{l}\text { Quartz, ferroan } \\
\text { calcite, kaolinite } \\
\text { and illite }\end{array}$} & \multirow{3}{*}{$\begin{array}{l}\text { Porosity is good and permeability is } \\
\text { poor to moderate. Secondary porosity is } \\
\text { patchy developed, after dissolution of } \\
\text { calcite cement. The increase in } \\
\text { abundance of fibrous illite, and resultant } \\
\text { microporosity development is primarily } \\
\text { responsible for poor permeability. }\end{array}$} \\
\hline & 2704.00 & 8.20 & 9.40 & 1.10 & & \\
\hline & 2705.00 & 9.80 & 10.80 & 0.60 & & \\
\hline & 2721.00 & 16.70 & 17.20 & 12.80 & \multirow{3}{*}{$\begin{array}{l}\text { Non-ferroan calcite, } \\
\text { illite and kaolinite }\end{array}$} & \multirow{3}{*}{$\begin{array}{l}\text { Porosity and permeability are good. } \\
\text { Secondary porosity are well developed, } \\
\text { but permeabilities are restricted by } \\
\text { fibrous authigenic illite, quartz } \\
\text { overgrowths and, to a lesser } \\
\text { extent, calcite. }\end{array}$} \\
\hline & 2722.00 & 15.50 & 14.10 & 8.20 & & \\
\hline & 2724.00 & 16.80 & 11.60 & 12.10 & & \\
\hline & 2726.00 & 15.60 & 14.80 & 4.80 & \multirow{3}{*}{$\begin{array}{l}\text { Non-ferroan calcite, } \\
\text { illite and kaolinite }\end{array}$} & \multirow{3}{*}{$\begin{array}{l}\text { Porosity is good and permeability is } \\
\text { poor to moderate. Fibrous illite, } \\
\text { from grain alteration, has reduced the } \\
\text { secondary porosity to microporosity in } \\
\text { places, resulting in reduced permeability. }\end{array}$} \\
\hline & 2728.00 & 12.70 & 11.80 & 1.60 & & \\
\hline & 2730.00 & 8.10 & 10.20 & 2.80 & & \\
\hline
\end{tabular}


Table 8. Cont.

\begin{tabular}{|c|c|c|c|c|c|c|}
\hline Borehole & Depth (m) & $\begin{array}{c}\text { Gas Expansion } \\
\text { Porosity }(\%)\end{array}$ & $\begin{array}{l}\text { Point Count } \\
\text { Porosity (\%) }\end{array}$ & $\begin{array}{c}\text { Klinkenberg } \\
\text { Corrected } \\
\text { Permeability }(\mathrm{mD})\end{array}$ & $\begin{array}{l}\text { Dominant Cement } \\
\text { under SEM and } \\
\text { Petrography }\end{array}$ & $\begin{array}{l}\text { Effect of Diagenesis on the } \\
\text { Reservoir Properties }\end{array}$ \\
\hline \multirow{4}{*}{ E-BA1 } & 2828.05 & 10.50 & 12.90 & 43.00 & \multirow[t]{2}{*}{$\begin{array}{l}\text { Quartz, kaolinite } \\
\text { and calcite }\end{array}$} & \multirow{2}{*}{$\begin{array}{l}\text { Porosity is good and permeability is } \\
\text { moderate to good. Quartz commonly } \\
\text { fills the secondary porosity and the } \\
\text { pseudomatrix results in it having } \\
\text { poorer permeability }\end{array}$} \\
\hline & 2832.92 & 11.00 & 12.30 & 11.70 & & \\
\hline & 2836.01 & 9.10 & 13.90 & 0.33 & \multirow[t]{2}{*}{ Quartz and kaolinite } & $\begin{array}{l}\text { Porosity is moderate with poor } \\
\text { permeability. Quartz overgrowths and } \\
\text { pseudomatrix are the most abundant } \\
\text { pore filling phases with lesser kaolinite. } \\
\text { Microporosity through pseudomatrix }\end{array}$ \\
\hline & 2842.10 & 10.50 & 11.40 & 3.21 & & restricts permeability. \\
\hline \multirow{7}{*}{ E-BB1 } & 2538.05 & 12.30 & 12.10 & 1.50 & \multirow[t]{4}{*}{$\begin{array}{l}\text { Quartz overgrowth } \\
\text { and non-ferroan } \\
\text { calcite }\end{array}$} & \multirow{4}{*}{$\begin{array}{l}\text { Good porosity and poor permeability. } \\
\text { Secondary porosity is good and } \\
\text { developed by leaching of calcite cement } \\
\text { Porosity is substantially reduced by } \\
\text { quartz overgrowths and compaction of } \\
\text { claystone clasts to form pseudomatrix. } \\
\text { Permeability is restricted by quartz } \\
\text { overgrowths and the development of } \\
\text { fibrous authigenic illite, } \\
\text { creating microporosity. }\end{array}$} \\
\hline & 2546.00 & 13.90 & 10.30 & 0.30 & & \\
\hline & 2554.40 & 11.70 & 14.10 & 0.28 & & \\
\hline & 2659.05 & 11.60 & 11.00 & 0.93 & & \\
\hline & 2663.18 & 2.80 & 3.70 & 0.01 & $\begin{array}{c}\text { Quartz and } \\
\text { non-ferroan calcite }\end{array}$ & $\begin{array}{l}\text { Poor porosity and permeability. } \\
\text { Porosity is reduced by pore filling } \\
\text { quartz, quartz overgrowths, calcite and } \\
\text { illite are the factors resulting in the } \\
\text { poor permeability. }\end{array}$ \\
\hline & 2720.55 & 14.30 & 11.60 & 11.20 & $\begin{array}{l}\text { Quartz, illite } \\
\text { and kaolinite }\end{array}$ & $\begin{array}{l}\text { Porosity is good and permeability is } \\
\text { poor to good. Quartz overgrowths } \\
\text { reduce intergranular porosity. Illite also } \\
\text { restricts permeability by }\end{array}$ \\
\hline & 2722.55 & 13.20 & 15.30 & 1.30 & & creating microporosity. \\
\hline
\end{tabular}


Table 8. Cont.

\begin{tabular}{|c|c|c|c|c|c|c|}
\hline Borehole & Depth (m) & $\begin{array}{l}\text { Gas Expansion } \\
\text { Porosity }(\%)\end{array}$ & $\begin{array}{l}\text { Point Count } \\
\text { Porosity }(\%)\end{array}$ & $\begin{array}{c}\text { Klinkenberg } \\
\text { Corrected } \\
\text { Permeability }(\mathrm{mD})\end{array}$ & $\begin{array}{l}\text { Dominant Cement } \\
\text { under SEM and } \\
\text { Petrography }\end{array}$ & $\begin{array}{l}\text { Effect of Diagenesis on the } \\
\text { Reservoir Properties }\end{array}$ \\
\hline & 2849.05 & 12.60 & 13.50 & 28.10 & \multirow{3}{*}{$\begin{array}{l}\text { Quartz overgrowth, } \\
\text { illite and kaolinite }\end{array}$} & $\begin{array}{l}\text { Porosity and permeability are } \\
\text { generally good. }\end{array}$ \\
\hline & 2852.03 & 8.00 & 7.20 & 0.09 & & $\begin{array}{l}\text { Porosity is good and permeability is } \\
\text { poor. The increase in the amounts of } \\
\text { illite and kaolinite and the presence of } \\
\text { pseudomatrix accounts for the }\end{array}$ \\
\hline & 2856.05 & 11.00 & 10.60 & 2.60 & & poor permeabilities. \\
\hline & 2872.05 & 11.90 & 8.90 & 16.50 & \multirow[t]{2}{*}{$\begin{array}{l}\text { Quartz, ferroan calcite } \\
\text { and pyrite }\end{array}$} & $\begin{array}{l}\text { Porosity and permeability are generally } \\
\text { good. Quartz and calcite commonly fills } \\
\text { the secondary porosity and the }\end{array}$ \\
\hline & 2894.05 & 11.70 & 10.60 & 12.80 & & having these porosity and permeability \\
\hline & 3280.06 & 5.40 & 13.60 & 0.01 & \multirow[t]{3}{*}{$\begin{array}{l}\text { Ferroan calcite, quartz } \\
\text { and illite }\end{array}$} & $\begin{array}{l}\text { Porosity is moderate to good and } \\
\text { permeability is generally poor. The very } \\
\text { poor porosity due to cementation by } \\
\text { calcite, quartz and illite. }\end{array}$ \\
\hline & 3284.87 & 11.30 & 7.50 & 1.20 & & The permeability is restricted by \\
\hline & 3290.31 & 10.60 & 12.10 & 0.02 & & overgrowth cementation. \\
\hline \multirow{5}{*}{ E-D3 } & 3268.58 & 1.50 & 3.50 & 0.00 & \multirow{2}{*}{$\begin{array}{l}\text { Kaolinite, illite, } \\
\text { non-ferroan calcite } \\
\text { and quartz } \\
\text { overgrowth }\end{array}$} & \multirow{2}{*}{$\begin{array}{l}\text { Porosity and permeability are very poor } \\
\text { Intergranular porosity is reduced by } \\
\text { quartz overgrowths and non-ferroan } \\
\text { calcite. Kaolinite, calcite and illite are } \\
\text { abundant, producing microporosity, and } \\
\text { hence reducing porosity permeability. }\end{array}$} \\
\hline & 3527.50 & 2.00 & 1.90 & 0.00 & & \\
\hline & 3733.25 & 16.00 & 14.30 & 5.80 & \multirow[t]{3}{*}{$\begin{array}{l}\text { Quartz overgrowth, } \\
\text { non-ferroan calcite } \\
\text { and pyrite }\end{array}$} & \multirow{3}{*}{$\begin{array}{l}\text { Porosity and permeability are good. } \\
\text { Quartz overgrowths and calcite have } \\
\text { reduced intergranular porosity. } \\
\text { Secondary porosity is developed by } \\
\text { leaching of calcite cement, but is } \\
\text { commonly reduced to microporosity by } \\
\text { authigenic illite. }\end{array}$} \\
\hline & 3736.03 & 10.00 & 11.80 & 7.90 & & \\
\hline & 3737.00 & 9.00 & 5.40 & 6.10 & & \\
\hline
\end{tabular}




\section{Discussion}

\subsection{Sandstone Classification}

The most widely used and effective methods for classifying sandstones combines the textural criterion (i.e., proportion of the matrix) with the compositional and mineralogical criteria $[44,45,47-52]$. In this study, the classification of the Bredasdorp sandstones is based on the classification schemes proposed by [47,50-52]. The aforementioned classification schemes show that the studied sandstones could be classified as subarkose and sub-lithic arenite (Figure 8).
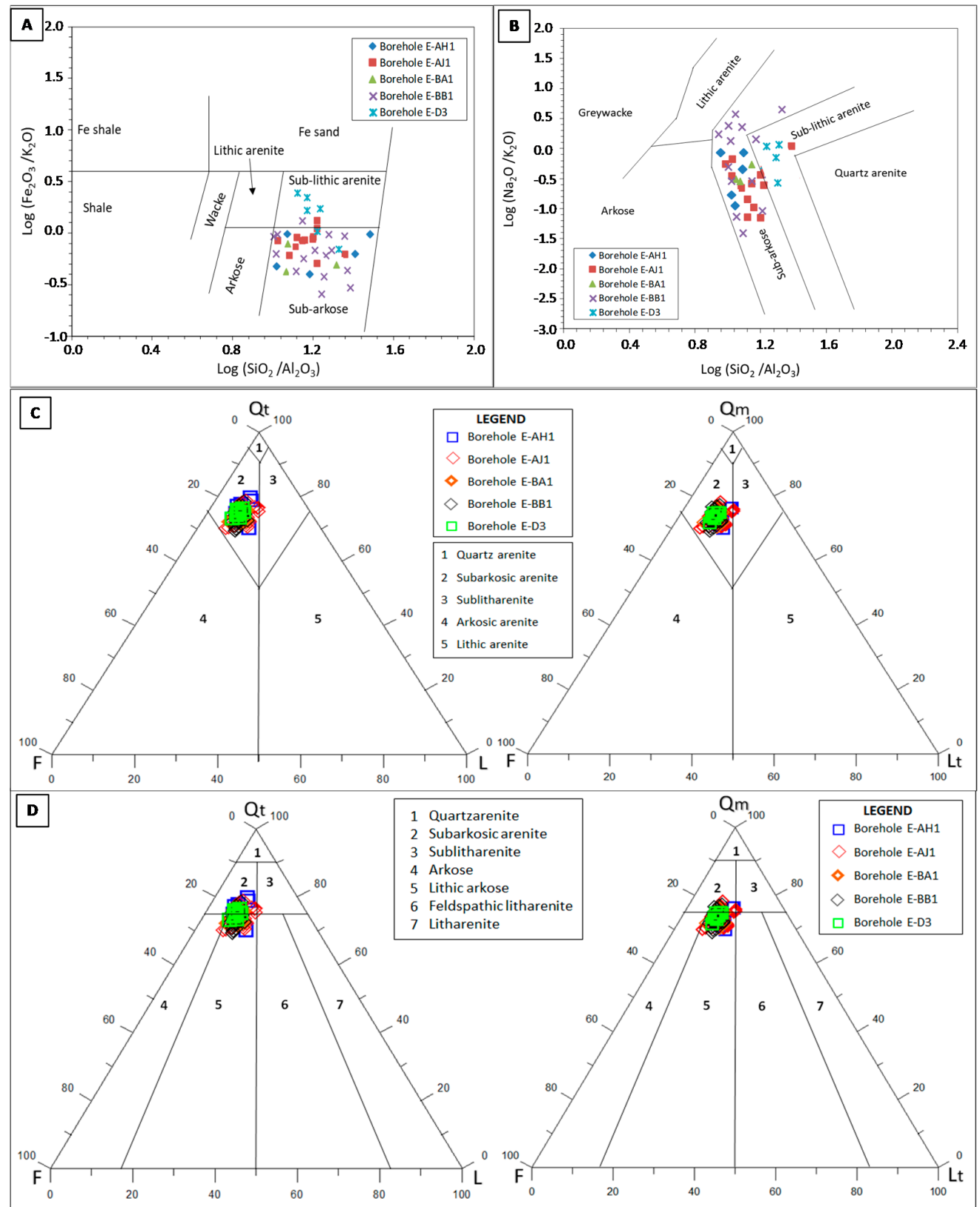

Figure 8. Classification of the Bredasdorp sandstones based on: (A) Binary plot of Log $\left(\mathrm{Na}_{2} \mathrm{O} / \mathrm{K}_{2} \mathrm{O}\right.$ ) versus $\mathrm{Log}\left(\mathrm{SiO}_{2} / \mathrm{Al}_{2} \mathrm{O}_{3}\right)$ (after [51]); (B) Binary plot of $\mathrm{Log}\left(\mathrm{Fe}_{2} \mathrm{O}_{3} / \mathrm{K}_{2} \mathrm{O}\right)$ versus $\log$ $\left(\mathrm{SiO}_{2} / \mathrm{Al}_{2} \mathrm{O}_{3}\right.$ ) (after [52]); (C) Ternary plot of total quartz-feldspar-lithic fragments (Qt-F-L) and monocrystalline quartz-feldspar-total lithic fragments (Qm-F-Lt) (after [47]); (D) Ternary plot of total quartz-feldspar-lithic fragments (Qt-F-L) and monocrystalline quartz-feldspar-total lithic fragments (Qm-F-Lt) (after [50]). 


\subsection{Diagenetic Processes and Stages}

The diagenetic identified textures and structures are grouped into early diagenesis, burial diagenesis and late (uplift-related) diagenesis. The summary of the diagenetic processes and changes in the sandstones of the Bredasdorp Basin is presented in Table 9. The key factors that affect the early diagenetic stage are geochemistry of the pore water, original mineralogy, and the presence of petroleum-related fluids. In the studied sandstones, the observed early diagenetic processes include precipitation of clay minerals (kaolinite, illite, smectite and chlorite), formation of carbonate (calcite) and pyrite cements, and glauconite as well as early dissolution of grain and minor compaction as a result of increasing overburden. The precipitation of pyrite, and alteration of glauconite pellets results from reducing conditions in marine pore fluids; this occurs shortly after burial. Accumulation of bacterial end-products in pore fluids results in precipitation of the carbonate minerals.

Table 9. Generalized paragenetic sequence of the Bredasdorp sandstones as established in this study.

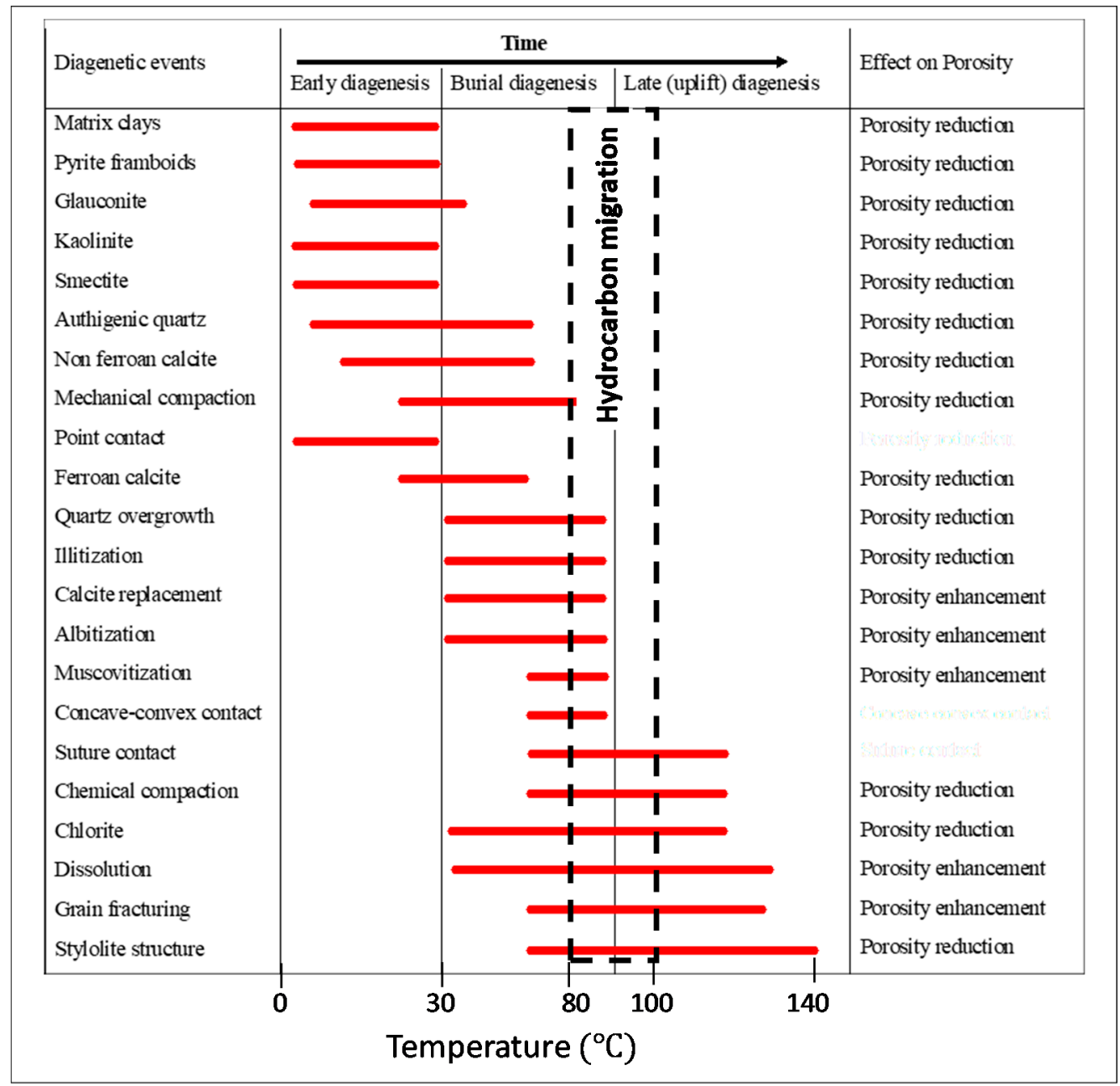

Note: The inserted temperature was extracted from [41].

Burial diagenesis in the sandstones started with the mechanical compaction of sands due to increasing overburden pressure. Mechanical compaction brought about the close packing of the detrital framework grains and resulted in the partial loss of porosity. In addition, this compaction is evidenced by long, concave-convex, sutured contacts existing between the framework grains. Progressive increase in burial depth due to overburden led to increase in temperature and pressure, resulting in the partial dissolution of feldspars and recrystallization of fine matrix. This feldspar dissolution is common in the Bredasdorp sandstones and it is caused by a relatively acidic condition, in which the stability of 
feldspars is reduced. Minerals that precipitated out of solution are typically authigenic pore-filling clays (i.e., kaolinite and chlorite), grain-coating clays (i.e., smectite, illite and smectite-illite mixed layers) and minor calcite cements. Early cementation of illite, smectite, chlorite and pyrite as well as compaction and quartz overgrowths could have formed due to the mobilization of ferrous iron from siderite and pyrite and by precipitation of ferric hydroxide under oxidizing ground-water conditions. The occurrence of authigenic, pore-filling kaolinite could probably be related to feldspar/glauconite alteration. The formation of kaolinite is dependent on: (1) the availability of adequate porosity and permeability to permit the movement of interstitial pore waters as well as to provide growth space, (2) Presence of $\mathrm{K}$-feldspar and/or muscovite as a source of $\mathrm{Al}$ and $\mathrm{Si}$, as well as the availability of pore waters of an acidic $\mathrm{pH}[2,5,6,13]$. The presence of altered $\mathrm{K}$-feldspar and muscovite in the sandstones shows that these minerals serve as sources of silica and aluminium and led to the formation of kaolinite. Generally, illite formation (illitization) typically happened after the precipitation of kaolinite and smectite which requires high potassium-pore water to achieve under elevated temperature $\left(90-130^{\circ} \mathrm{C}\right)[2,3,6,12]$. The development of illite needs a growth medium (pore fluid and space) that has potassium $(\mathrm{K})$, silica $(\mathrm{Si})$ and aluminium $(\mathrm{Al})$ compositions $[3,6,7]$. The EDX graph presented in Figure 4 shows presence of silica, aluminium, potassium, iron and magnesium elements which concur with the chemical composition of illite $\left(\left(\mathrm{K}, \mathrm{H}_{3} \mathrm{O}\right)(\mathrm{Al}, \mathrm{Mg}, \mathrm{Fe})_{2}(\mathrm{Si}, \mathrm{Al})_{4} \mathrm{O}_{10}\left[(\mathrm{OH})_{2},\left(\mathrm{H}_{2} \mathrm{O}\right)\right]\right)$. SEM observation shows that illite post-dates the silica cementation.

The chemical composition of smectite shows that smectite is made up of significant amount of elements like $\mathrm{Al}, \mathrm{Fe}, \mathrm{Mg}, \mathrm{Si}, \mathrm{Ca}$ and $\mathrm{Na}$. During the transformation of smectite to illite or destruction of the mixed illite-smectite interlayers during illitization, substantial amounts of these elements can be released, depending on the original composition of the rock. According to [3], smectite is recrystallized to illite through the process of illitization, wherein potassium $\left(\mathrm{K}^{+}\right)$is added to the mixed interlayer space and the amount of the aluminium tetrahedral $\left(\mathrm{Al}^{3+}\right)$ have to be increased for the conversion or transformation of smectite to illite (Smectite $+\mathrm{Al}^{3+}+\mathrm{K}^{+}=$Illite $+\mathrm{Si}^{4+}$ ) to occur [5,6]. This is supported or in agreement with the EDX graph presented in Figure 4. The released silica $\left(\mathrm{Si}^{4+}\right)$ is thought to have formed or perhaps add to the quartz cement in the sandstones, while the $\mathrm{Fe}$ and $\mathrm{Mg}$ are alleged to have being formed as part of reaction products that are more stable under the increased burial temperature conditions [3]. The formation of chlorite mineral could be related to the reactions of dioctahedral clay mineral (i.e., kaolinite) with carbonates [5,6]. The growth of calcite, illite and chlorite all reduces the porosity of the reservoir interval. The pore filling clays that precipitated into secondary pores may have been formed as a result of the dissolution of feldspars. These clays as well as other cements led to the reducing secondary porosity in the burial diagenesis. Stylolites and sutured contacts are evidence of chemical compaction causing pressure dissolution. Most of the observed secondary intergranular pores were created by the dissolution of detrital feldspar and carbonate cement. Also, kaolinite is formed during the late dissolution of feldspar grains by organic acids generated during kerogen maturation.

In the late diagenetic stage, minerals that were originally formed under high temperature and pressure in the burial diagenetic stage become unstable and are replaced or altered to more stable mineral. Despite the fact that the pore waters in the late diagenetic stage are of low ionic strength, they can still result in substantial mineralogical changes, such as the alteration feldspars to clay minerals, especially kaolinite [6]. Also, previously formed cements (i.e., carbonate) are dissolved (decementation), resulting in porosity increase. Some of the observed kaolinites are formed because of weathering and replacement of feldspars. The kaolinitization of feldspars was as a result of the presence of fresh surface-water at uplift. Calcite and kaolinite were also partially dissolved and leached, which led to the formation of secondary porosity. Chemical compaction started at this stage and occurred by pressure dissolution along intergranular contacts and fractures, providing silica for quartz cementation. The precipitation of secondary silica was accompanied, and most likely followed by partial or complete dissolution of the carbonate cements. Though, it is challenging to define the exact timing and burial depth of diagenetic alterations, a general paragenetic sequence can be reconstructed and established by synthesizing the petrologic features, burial and thermal histories and various 
diagenetic minerals and features. The generalized paragenetic sequence and relative timing of the major diagenetic events of the Bredasdorp sandstones is shown in Table 9.

\subsection{Reservoir Properties}

The relationship and percentages of porosity and permeability are the main properties that determine the quality of a reservoir rocks. These properties play a vital role in the diagenesis of sediments by controlling the flow of fluids through them. The fluid that flows in the sandstone causes dissolution, cementation, and authigenesis of minerals. To some extent, porosity and permeability are influenced by the textural characteristics of grain size, shape, packing, and arrangement. The point count porosity and gas expansion porosity are highest in exploration well E-AH1 and lowest in exploration well E-D3. Specifically, the gas expansion porosity for exploration wells E-AH1, E-AJ1, E-BA1, E-BB1 and E-D3 range between 14.2-20.6\%, 6.7-16.8\%, 9.1-11\%, 2.8-14.3\%, and 1.5-16\%, respectively. The permeability values range between $22-61 \mathrm{mD}, 0.6-19.4 \mathrm{mD}, 0.3-11.7 \mathrm{mD}, 0.01-28.1 \mathrm{mD}$, and 0.01-7.9 mD for exploration wells E-AH1, E-AJ1, E-BA1, E-BB1 and E-D3, respectively (Figure 7). Just like the point count porosity, the gas expansion porosity and Klinkenberg corrected permeability are highest in exploration well E-AH1 and lowest in exploration well E-D3. Borehole E-AH1 is mostly made up of subarkosic sandstones, while Borehole E-D3 is consists of lithic arkose. Generally, the arkosic sandstones have higher percentages of porosity and permeability. These sandstone types could have reacted to diagenetical processes differently, hence resulting in the variation in their reservoir properties. These variations in the porosity and permeability shows the variety of different diagenetic processes that acted to greater or lesser extent on the sandstones in the different boreholes and under different conditions. For example, mechanical compaction causes sandstones with more ductile grains to lose permeability as compared to sandstones with less ductile grains. There is no particular order or pattern of increasing or decreasing of porosities and permeability with depth (Figure 9). The various controls (i.e., detrital matrix and cements) on reservoir quality acted differently in each borehole and at different depths and sand units. The main diagenetic process that has significantly affected the reservoir properties of the sandstones is cementation by clay minerals. Sandstones at shallow depth are expected to be more porous compared to deeply buried sand units, however, the opposite is seen in most of the studied boreholes. For example, in boreholes E-D3, the porosity of the sand unit is $2-3.5 \%$ at a depth of about $3268 \mathrm{~m}$, but decreases to $1.5-2 \%$ at a depth of approximately $3527 \mathrm{~m}$, perhaps pointing to the influence of compaction as a result of increased overburden or burial depth. On the contrary, this porosity (1.5-2\%) at a depth of about $3527 \mathrm{~m}$ increases to $12.5-15 \%$ at a depth of approximately $3733 \mathrm{~m}$ and suddenly reduced to $3-4 \%$ at a depth of around $3740 \mathrm{~m}$. Petrographic and SEM observations of the sandstones from the depths of 3733-3740 $\mathrm{m}$ show that secondary porosity is developed in the sandstones by leaching of calcite cement and dissolution of feldspar. However, this porosity is reduced to microporosity by authigenic illite at the depth of about $3740 \mathrm{~m}$. In Boreholes E-AJ1 and E-BB1, the influence of compaction and cementation is complex with no particular pattern, perhaps suggesting that diagenesis is the main challenge to reservoir characterization in the Bredasdorp Basin. Facies variance with depth in the boreholes and depositional environment (i.e., distal and proximal areas) could also have affected the reservoir properties of the individual boreholes. However, proximal and distal areas, reworking and facies variations have not been interpreted or evaluated in this study. 


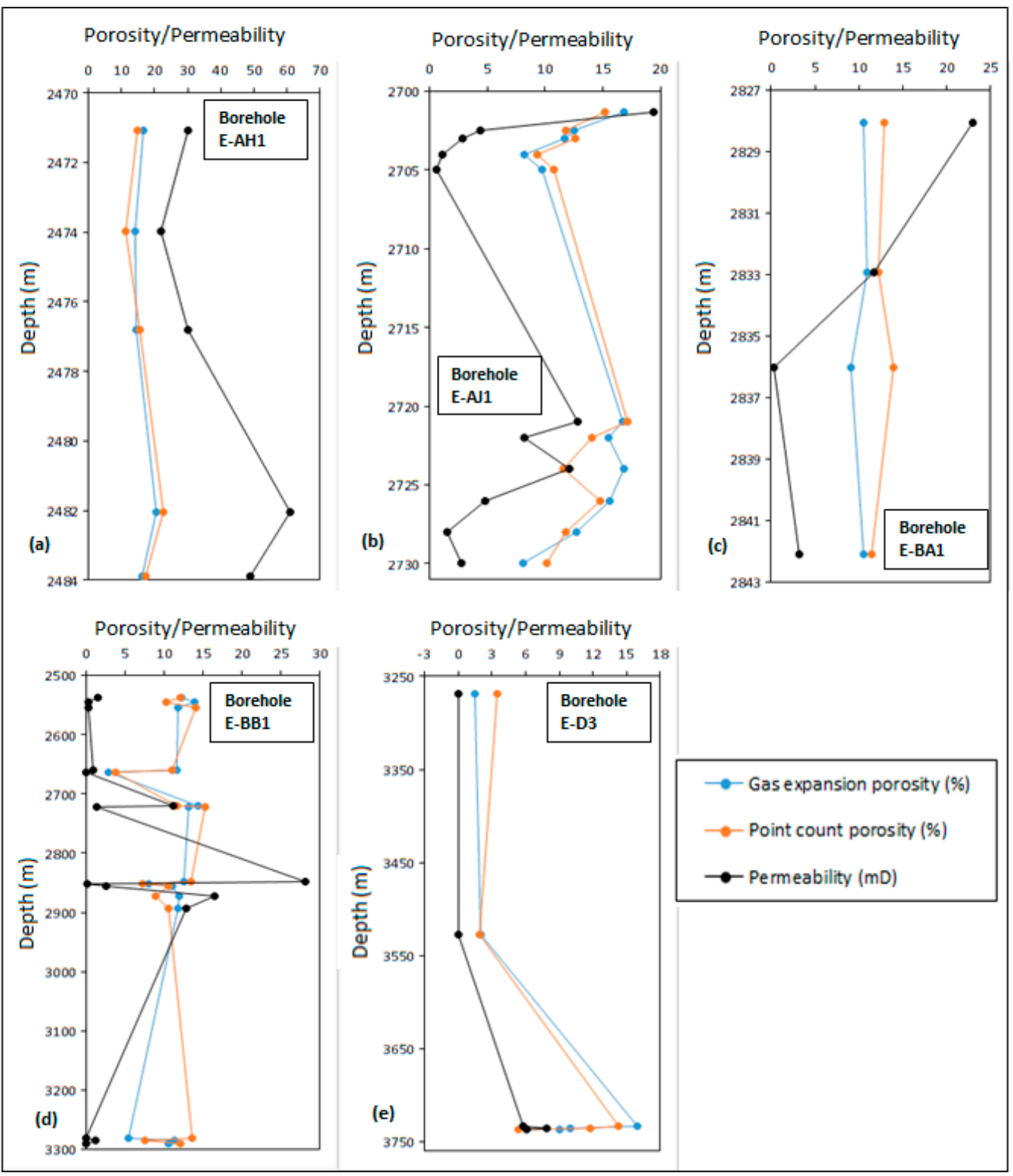

Figure 9. Downhole point count porosity, Poroperm Porosity and permeability for exploration wells: (a) E-AH1; (b); E-AJ1; (c); E-BA1; (d) E-BB1; (e) E-D3.

The percentage of detrital matrix and cements in Boreholes E-AH1, E-AJ1, E-BA1, E-BB1 and E-D3 varies between $8.8-20.2 \%$, 8.6-15.2\%, 7.6-16\%, 10.4-20.6\% and $12.4-16.8 \%$, respectively, in no particular order with increasing burial depth. This possibly indicate that diagenesis is the main control on reservoir properties. Although, both primary and secondary pore types are present in the sandstones at different depth intervals, the extensive matrix in some of the samples hinders cementation by inhibiting nucleation of overgrowths. Petrographic and SEM examinations of the sandstones revealed that areas (depths) with less clay minerals are often associated with high porosity and permeability, and vice versa. The areas with high permeability values generally have significant preservation of primary porosity and/or extensive development of secondary porosity. In samples where large, isolated secondary pores occurred as a result of grain and/or cement dissolution, often give rise to high porosity and somewhat less permeability. The petrographic studies revealed a number of diagenetic controls on porosity development, which include cementation and compaction. It also shows that the porosity increases in samples of floating to point contacts and decreasing with long, concave-convex and sutured contacts (Figure 7). A scatter plot of point counted porosity and gas expansion porosity against permeability 
show a positive correlation (Figure 10). Also, binary plot of the point count porosity versus gas expansion porosity have a direct or positive correlation, suggesting that the porosity values are reliable. Silica, carbonate and authigenic clay cements are the main cements that affected the reservoir properties of the Bredasdorp sandstones. In order to statistically investigate these controls on porosity, the sum of cements and authigenic clays were plotted against point counted porosity, gas expansion porosity and permeability and they all have a relatively good inverse correlation (Figure 10). This negative or inverse correlation point to the fact that these controls are jointly the main ones of concern for reservoir quality prediction. Carbonate cement, especially the poikilitopic calcite tends to assemble in pores, blocking pore throats. Hence, it reduces permeability more than grain coating cements like quartz overgrowth.
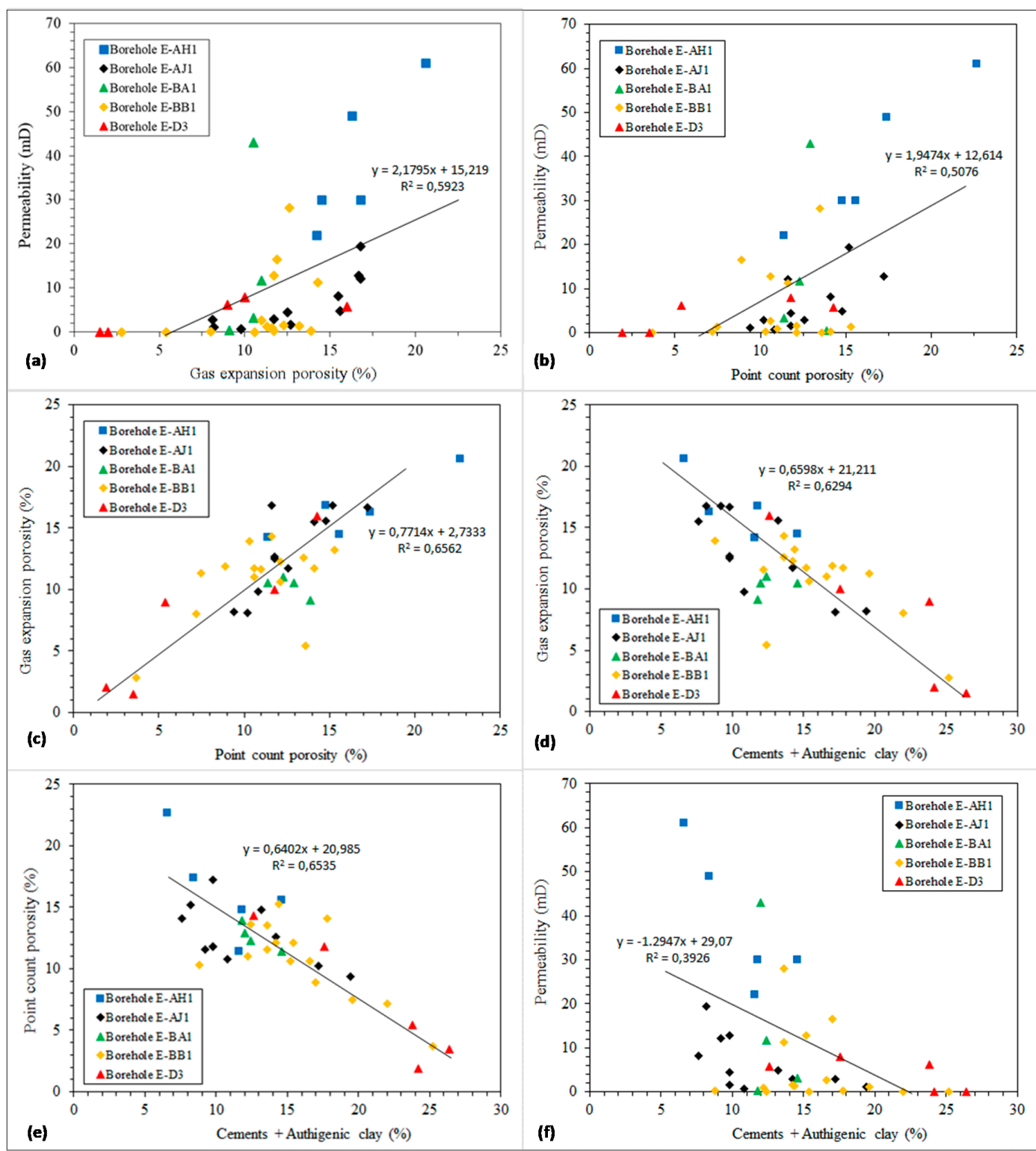

Figure 10. Scatter plots of the reservoir property data of the Bredasdorp sandstones showing the relationship between visual point counted porosity, gas expansion porosity, permeability, and cements plus authigenic clays: (a) Gas expansion porosity versus permeability; (b) Point counted porosity against permeability; (c) Point counted porosity versus gas expansion porosity; (d) Cements + authigenic clays against gas expansion porosity; (e) Cements + authigenic clays versus point counted porosity; (f) Cements + authigenic clays against permeability. 


\subsection{Implications of Diagenesis for Reservoir Quality}

The diagenetic environment of the Bredasdorp Basin was constantly changing, resulting in the complex diagenetic phenomena in the present reservoirs. Diagenesis modifies the original pore type and geometry of sandstones and hence controls its final porosity and permeability. A good knowledge or interpretation of the diagenetic history as a product of depositional environments, sediment composition, and fluid migration pattern is very important when predicting the reservoir quality of sandstones [31,41,43]. Reservoir quality of the Bredasdorp sandstones is chiefly controlled by diagenetic processes that either reduce or increase porosity and permeability. The most important diagenetic processes that affected the reservoir quality of the Bredasdorp sandstones are mechanical compaction, cementation by carbonate (calcite), glauconite, silica (quartz), sulphide (pyrite) and authigenic clay (kaolinite, illite, smectite and chlorite) and dissolution of minerals (i.e., feldspar). The inferred potential reservoir quality of the Bredasdorp sandstones is presented in Figure 11. As the deposited sands become compacted as a result of overburden, grain contact changes from point contacts to sutured contact due to progressive burial, which resulted in the reduction of primary intergranular porosity and pore radii.

The primary porosities are the abundant pore types, however, they are reduced as a result of calcite cementation, formation of authigenic clays and compaction. The most abundant authigenic clay in the studied sandstones is kaolinite and it occurs as rims around detrital grains as well as pore-lining and pore- filling cements. This clay acts as pore choking cement, which reduces porosity and creates permeability barrier and may present irreducible water saturation traps. Kaolinite infilling pores, while apparently reducing porosity, preserves a substantial microporosity. The main issue with kaolinite is that the individual plates are susceptible to become removed during oil production, and block the pore-throats, with a major reduction in permeability [37]. Also, the amount or proportion of fibrous illite in the analysed sandstones are small, hence it has little effect in destroying permeability of the sandstones. Kaolinite and mixed layer clays caused reduction in primary porosity, but can also form and preserve microporosity among kaolinite crystals. The early formed non-ferroan calcite cement pervasively fill in primary porosity and occlude pore throat, while the ferroan calcite cements fill in residual primary porosity as well as secondary porosity created by the dissolution of feldspars [31]. Silica cements often destroy primary porosity and their presence in the sandstones could probably hinder the preservation of primary porosity. Quartz overgrowths in the sandstones coat the detrital quartz grains and led to the decrease of pore radii, resulting in the reduction of porosity and permeability. Pyrite cement is present in minor proportions but to some extent, locally restricts the pore geometry and pore size distribution.

Reservoir quality of the sandstones has been improved to various extents due to the existence of secondary porosity developed by the dissolution of feldspars and carbonate cements. The observed secondary porosities in the sandstones are secondary intragranular pores, dissolution pores and fractured pores. Secondary intragranular and dissolution pores are created as a result of the dissolution of detrital feldspar grains, dissolution of clay minerals formed by alteration of the pseudomatrix and from the dissolution of carbonate cement. Likewise, the fractured pores are due to structural forces and differential compaction. Generally, porosities in the sandstones are significantly reduced by pseudomatrix and silica cements. Permeability has been restricted by the development of fibrous authigenic illite, quartz overgrowths and, to a lesser extent, carbonate and pyrite cements. Most of the samples with poor or low porosity and permeability values have undergone pressure solution along carbonaceous streaks, resulting in more extensive quartz and kaolinite cementation. Also, deeper samples with poor porosities are characterized by pervasive silica cements and development of pseudomatrix, hence, the development of secondary porosity is very low or absent. Despite the formation of secondary porosity, diagenesis has significantly reduced the reservoir quality of the Bredasdorp sandstones. The level or degree of impact of diagenesis on the reservoir quality varies differently in each borehole and at different depths and sand units. Also, the intermittent introduction of 
increased and decreased temperatures followed by decreased temperatures has affected the diagenesis of the basin [33].

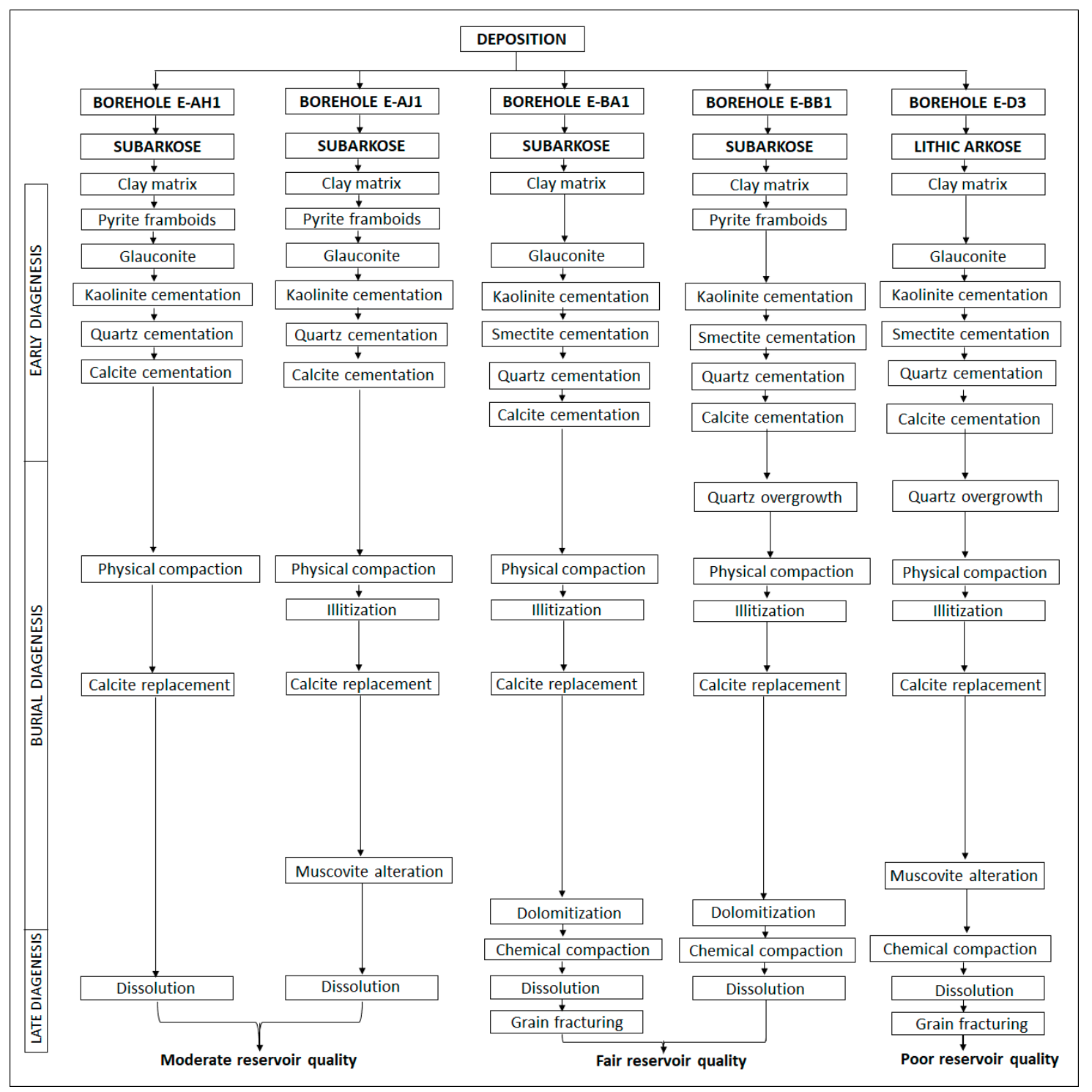

Figure 11. Flow chart of the main diagenetic processes in Bredasdorp sandstones and their inferred potential reservoir quality.

Generally, the studied boreholes are located in a relatively similar basinal setting with the Oribi and Oryx oil fields, and they are drilled to test for hydrocarbons within domal structural and stratigraphic closure at various target levels [31]. The primary target is the stratigraphically trapped sandstones within the 13 At1 to 14 At1 (mid-Aptian) interval [31,41]. These sandstones are stacked mass-flows deposited within a lowstand wedge. The studied boreholes were reported to have the same or similar structural elements and paleo-geothermal gradient and fluid compositions when hydrocarbon were introduced [31-33,37]. The total organic carbon (TOC), vitrinite reflectance and Tmax values for the studied samples are relatively variable but still comparable with those obtained for boreholes within the Oribi and Oryx oil fields, perhaps indicating the different source rock in the basinal setting [31,41]. Acho [41] indicated that wells E-AH1, E-AJ1 and E-BB1 are stratigraphically situated in the organic-rich mudstone/shale source rocks, thus there was the formation and accumulation of hydrocarbons. On the other hand, wells E-BA1 and E-D3 are stratigraphically located relatively further away from the organic-rich mudstone/shale source rocks and as a result shows low hydrocarbons potentials. Also, 
diagenesis and fault-control of sedimentation may to have affected the capacity of the boreholes to retain significant amount of the generated hydrocarbons. Likewise, the age, transportation, deposition and thermal history of sediment in the basin, may have contributed or influenced the potential and type of hydrocarbon formation [37].

\section{Conclusions}

The Bredasdorp sandstones are predominantly fine-grained, and moderately-well sorted, and their mineral composition of classification corresponds to subarkosic and lithic arkose. The sandstones have considerable heterogeneity with a wide range of porosity from 14.2 to $20.6 \%, 6.7$ to $16.8 \%$, 9.1 to $11 \%, 2.8$ to $14.3 \%$, and 1.5 to $16 \%$ in exploration wells E-AH1, E-AJ1, E-BA1, E-BB1 and E-D3, respectively. The permeability varies from 22 to $61 \mathrm{mD}, 0.6$ to $19.4 \mathrm{mD}, 0.3$ to $11.7 \mathrm{mD}$, 0.01 to $28.1 \mathrm{mD}$, and 0.01 to $7.9 \mathrm{mD}$ in wells E-AH1, E-AJ1, E-BA1, E-BB1 and E-D3, respectively. These sandstones show good reservoir characteristics in some intervals due to high porosity and permeability, while in some other intervals they show fair to bad reservoir characteristics due to low porosity and permeability. The high reservoir quality in the Borehole E-AH1 and E-D3 is due to diagenetic processes (i.e., dissolution, leaching and grain fracturing) that enhanced both the porosity and permeability. In contrast, the poor reservoir quality in the Borehole E-D3 is a result of the prevalence of cementation by authigenic clays and compaction (i.e., concavo-convex and suture contacts). The various controls on reservoir quality act differently in each borehole and at different depths and sand units. Hydrocarbon generation and migration has influenced some diagenetic effects on cements and clays. Cementation by kaolinite, illite, illite-smectite, quartz, calcite and pore-filling chlorite are the main factors for the deterioration of reservoir quality. The influence of cementation and compaction is complex with no particular pattern with increasing depth, suggesting that diagenesis is the main challenge to reservoir characterization in the Bredasdorp Basin. In addition, compaction also account for reservoir quality reduction. In general, the primary porosity of the sandstones is influenced by the filling of clay matrix, early authigenic minerals of pyrite and glauconite, and cements such as carbonate (calcite) and silica (quartz). The progressive burial and compaction also contribute to porosity reduction due to compaction as indicated by concave-convex and sutured contacts. In few samples, the early carbonate cement prevented or reduced further compaction, ensuing in close packing of clastic grains. Dissolution of metastable minerals, cements and leaching of the unstable grains led to secondary porosity developments in the sandstones. However, the solute is not transported over a large scale in the deep burial environment; as a result, simultaneous precipitation of byproducts of feldspar dissolution, such as authigenic illite, kaolinite and quartz cement, will occur in situ or in adjacent pores, which results in further heterogeneity of the reservoirs. In general, there is no particular diagenetic process that exclusively controls the type or form of porosity evolution in the sandstones. Instead, it seems that the main types of cements (clay minerals, calcite, and silica) and to some extent, compaction collectively controlled the reservoir quality of the sandstones. This study shows that paragenetic sequences and their relation to hydrocarbon generation and migration can be a valuable tool for oil migration into the reservoir sand units.

Supplementary Materials: The following are available online at http://www.mdpi.com/2075-163X/10/9/757/s1.

Author Contributions: Conceptualization, T.L.B. and K.L.; methodology, T.L.B., K.L. and C.B.; software, O.G. and C.B.; validation, T.L.B., K.L., O.G. and C.B.; formal analysis, T.L.B., K.L. and C.B.; investigation, T.L.B., K.L., O.G. and C.B.; resources, K.L. and O.G.; data curation, T.L.B., K.L. and C.B.; writing-original draft preparation, T.L.B.; writing-review and editing, T.L.B., K.L., O.G. and C.B.; visualization, T.L.B., K.L., O.G., and C.B; supervision, K.L. and O.G.; project administration, K.L. and O.G.; funding acquisition, K.L. All authors have read and agreed to the published version of the manuscript.

Funding: The authors are grateful to the National Research Foundation-Southern African Systems Analysis Centre (NRF-SASAC; UID: 118768) and DSI-NRF Centre of Excellence (CoE) for Integrated Mineral and Energy Resource Analysis (CIMERA) for financial support. 
Acknowledgments: The Petroleum Agency of South Africa (PASA) and the Govan Mbeki Research and Development Centre (GMRDC) of the University of Fort Hare are appreciated for granting access to the cores and logistic supports, respectively.

Conflicts of Interest: The authors declare no conflict of interest.

\section{References}

1. Bjorlykke, K.; Diagenesis, I. Development in Sedimentology; Chilingarian, G.V., Wolf, K.H., Eds.; Elsevier: Amsterdam, The Netherlands, 1988; Volume 41, pp. 555-588.

2. Zou, C.; Zhu, R.; Liu, K.; Su, L.; Bai, B.; Zhang, X.; Yuan, X.; Wang, J. Tight gas sandstone reservoirs in China: Characteristics and recognition criteria. J. Pet. Sci. Eng. 2012, 88, 82-91. [CrossRef]

3. Baiyegunhi, C.; Liu, K.; Gwavava, O. Diagenesis and reservoir properties of the Permian Ecca Group sandstones and mudrocks in the Eastern Cape Province, South Africa. Minerals 2017, 88, 1-26.

4. Bloch, S.; Lander, R.H.; Bonell, L. Anomalously high porosity and permeability in deeply buried sandstones reservoirs. Origin and predictability. AAPG Bull. 2002, 86, 301-328.

5. Burley, S.D.; Worden, R.H. (Eds.) Sandstone diagenesis: The evolution of sand to stone. In Sandstone Diagenesis Recent and Ancient; International Association of Sedimentologists Reprint Series; Blackwell Publishing: Malden, MA, USA, 2003; Volume 4, pp. 34-44.

6. Mackenzie, F.T. Diagenesis, and sedimentary rocks. In Treatise on Geochemistry, 2nd ed.; Elsevier: Oxford, UK, 2005; Volume 7, 446p.

7. Milliken, K.L. Understanding diagenetic controls on sandstone reservoir quality: A compendium of influential papers. In Getting Started, \#4; AAPG/Datapages: Tulsa, OK, USA, 2006.

8. Ajdukiewicz, J.M.; Lander, R.H. Sandstone reservoir quality prediction: The state of the art. AAPG Bull. 2010, 94, 1083-1091. [CrossRef]

9. Boggs, S., Jr. Petrology of Sedimentary Rocks, 2nd ed.; Cambridge University Press: Cambridge, UK, 2009; 600p.

10. Roswell, D.M.; De Swart, A.M.J. Diagenesis in Cape and Karroo sediments, South Africa, and its bearing on their hydrocarbon potential. S. Afr. J. Geol. 1976, 79, 81-145.

11. Reed, J.S.; Eriksson, K.A.; Kowalewski, M. Climatic, depositional and burial controls on diagenesis of Appalachian Carboniferous sandstones: Qualitative and quantitative methods. Sediment. Geol. 2005, 176, 225-246. [CrossRef]

12. Macquaker, J.H.S.; Taylor, K.G.; Keller, M.; Polya, D. Compositional controls on early diagenetic pathways in fine-grained sedimentary rocks: Implications for predicting unconventional reservoir attributes of mudstones. AAPG Bull. 2014, 93, 587-603. [CrossRef]

13. Chima, P.; Baiyegunhi, C.; Liu, K.; Gwavava, O. Diagenesis and rock properties of sandstones from the Stormberg Group, Karoo Supergroup in the Eastern Cape Province of South Africa. Open Geosci. 2018, 10, 740-771. [CrossRef]

14. Burden, P.L.A. Soekor, partners explore possibilities in Bredasdorp Basin off South Africa. Oil Gas J. 1992, 90, 109-112.

15. Brown, L.F.; Brink, G.J.; Doherty, S.; Jollands, A.; Jungslager, E.H.A.; Keenan, J.H.G.; Muntigh, A.; van Wyk, N.J.S. Sequence stratigraphy in offshore South African divergent basins: An atlas on exploration for cretaceous lowstand traps, by Soeker (Pty) Ltd. In AAPG Studies in Geology, \#41; AAPG/Datapages: Tulsa, OK, USA, 1995; pp. 83-131.

16. Jungslager, E.H.A. Geological Evaluation of the Remaining Prospectivity for Oil and Gas of the Pre-1At1 "Synrift" Succession in Block 9, Republic of South Africa. Unpublished SOEKOR Technical Report SOE-EXP-RPT-0380. 1996; 63p.

17. McMillan, I.K.; Brink, G.J.; Broad, D.S.; Maier, J.J. Late Mesozoic sedimentary basins off the south coast of South Africa. In Sedimentary Basins of the World—African Basins; Selley, R.C., Ed.; Elsevier Science Publisher B.V.: Amsterdam, The Netherlands, 1997; pp. 319-376.

18. Petroleum Geo-Services, PGS. Re-Evaluation of the F-A Field and Satellite; Petroleum Geo-Services, PGS: Cape Town, South Africa, 1999; 57p, Unpublished. 
19. Petroleum Agency South Africa, PASA. Petroleum Exploration in South Africa, Information and Opportunities; Online Report; Petroleum Agency South Africa PASA: Cape Town, South Africa, 2003; pp. 12-15. Available online: http://www.petroleumagencysa.com/images/pdfs/pet_expl_opp_2003fw.pdf (accessed on 17 November 2019).

20. Petroleum Agency South Africa, PASA. Petroleum Exploration in South Africa, Information and Opportunities; Online Report; Petroleum Agency South Africa PASA: Cape Town, South Africa, 2005; pp. 16-18. Available online: http://www.petroleumagencysa.com/images/pdfs/pet_expl_opp_2005fw.pdf (accessed on 17 November 2005).

21. Petroleum Agency South Africa, PASA. Petroleum Exploration in South Africa, Information and Opportunities; Online Report; Petroleum Agency South Africa PASA: Cape Town, South Africa, 2015; pp. 13-16. Available online: http://www.petroleumagencysa.com/images/pdfs/pet_expl_opp_2009fw.pdf (accessed on 17 November 2009).

22. Petroleum Agency South Africa, PASA. Petroleum Exploration in South Africa, Information and Opportunities; Online Report; PASA: Cape Town, South Africa, 2012; pp. 25-29. Available online: http://www. petroleumagencysa.com/images/pdfs/pet_expl_opp_2012fw.pdf (accessed on 17 November 2019).

23. Petroleum Agency South Africa, PASA. Petroleum Exploration in South Africa, Information and Opportunities; Online Report; Petroleum Agency South Africa PASA: Cape Town, South Africa, 2015; pp. 1-15. Available online: http://www.petroleumagencysa.com/images/pdfs/pet_expl_opp_2015fw.pdf (accessed on 17 November 2019).

24. Broad, D.S.; Jungslager, E.H.A.; McLachlan, I.R.; Roux, J. Offshore Mesozoic Basins. In The Geology of South Africa; Johnson, M.R., Anhaeusser, C.R., Thomas, R.J., Eds.; Geological Society of South Africa, Johannesburg/Council for Geoscience: Pretoria, South Africa, 2006; pp. 553-571.

25. Tinker, J.; de Wit, M.; Brown, R. Linking source and sink: Evaluating the balance between onshore erosion and offshore sediment accumulation since Gondwana break-up, South Africa. Tectonophysics 2008, 455, 94-103. [CrossRef]

26. Akinlua, A.; Sigedle, A.; Buthelezi, T.; Fadipe, O.A. Trace element geochemistry of crude oils and condensates from South African Basins. Mar. Pet. Geol. 2015, 59, 286-293. [CrossRef]

27. Liro, L.M.; Dawson, W.C. Reservoir systems of selected basins of the South Atlantic. In Petroleum System of South Atlantic Margins; Mello, M.R., Katz, B.J., Eds.; American Association of Petroleum Geologists: Tulsa, OK, USA, 2000; Volume 73, pp. 77-92.

28. Menger-Allogo, A. Sedimentology and Stratigraphy of Deep-Water Reservoirs in the 9A to 14A Sequences of the Central Bredasdorp Basin, Offshore South Africa. Master's Dissertation, Stellenbosch University, Stellenbosch, South Africa, 2006; 203p. Unpublished.

29. Dingle, R.V.; Siesser, W.G.; Newton, A.R. Mesozoic and Tertiary Geology of Southern African; American Association for the Advancement of Science: Washington, DC, USA, 1983; pp. 99-106.

30. De Wit, M.J.; Ransome, I.G. Regional inversion tectonics along the southern margin of Gondwana. In Proceedings of theInversion Tectonics of the Cape Fold Belt, Karoo and Cretaceous Basins of Southern Africa, Cape Town, South Africa, 2-6 December 1991; De Wit, M.J., Ransome, I.G., Eds.; CRC Press: Rotterdam, The Netherlands, 1992; pp. 15-22.

31. Davies, C.P.N. Unusual biomarker maturation ratio changes through the oil window, a consequence of varied thermal history. Org. Geochem. 1997, 27, 537-560. [CrossRef]

32. Duncan, R.A. Hotspots in the Southern Ocean, an absolute frame of reference for motion of the Gondwana continent. Tectonophysics 1981, 74, 29-42. [CrossRef]

33. Hartnady, C.J.H.; Le Roex, A.P. Southern Ocean hotspot tracks and the Cenozoic absolute motion of the African, Antarctic and South American plates. Earth Planet. Sci. Lett. 1985, 75, 245-257. [CrossRef]

34. Emery, K.O.; Uchapi, E.; Bowin, C.O.; Phillips, J.; Simpson, E.S.W. Continental margin of western Africa: Cape St Francis (South Africa) to Walvis Ridge (South West Africa). AAPG Bull. 1974, 59, 34-59.

35. Hartnady, C.J.H.; Partridge, T.C. Neotectonic uplift in Southern Africa: A brief review and geodynamic conjecture. In Proceedings of the Centernnial Geocongress Geological Society of South Africa, Johannesburg, South Africa, 3-7 April 1995; Volume 11, pp. 456-459.

36. Ben-Avraham, Z.; Hartnady, C.J.H.; Malan, J.A. Early tectonic extension between the Agulhas Bank and the Falkland Plateau due to the rotation of the Lafonia microplate. Earth Planet. Sci. Lett. 1993, 117, 43-58. [CrossRef] 
37. Broad, D.S.; Mills, S.R. South Africa offshore exploratory potential in variety of basins. Oil Gas J. 1993, $91,38-44$.

38. Sonibare, W.A. Structure and Evolution of Basin and Petroleum Systems within a Transform-Related Passive Margin Setting: Data-Based Insights from Crust-Scale 3D Modelling of the Western Bredasdorp Basin, Offshore South Africa. Ph.D. Thesis, Stellenbosch University, Stellenbosch, South Africa, 2015; pp. 16-44.

39. Peters, K.E.; Cassa, M.R. Applied source rock geochemistry. In The Petroleum System-From Source to Trap; Magoon, L.B., Dow, W.G., Eds.; American Association of Petroleum Geologists: Tulsa, OK, USA, 1994; Volume 60, pp. 93-120.

40. McCarthy, K.; Rojas, K.; Niemann, M.; Palmowski, D.; Peters, K.; Stankiewicz, A. Basic petroleum geochemistry for source rock evaluation. Oil Field Rev. 2011, 23, 32-43.

41. Acho, B.C. Assessing Hydrocarbon Potential in Cretaceous Sediments in the Western Bredasdorp Sub-Basin in the Outeniqua Basin South Africa. Master's Dissertation, University of Western Cape, Cape Town, South Africa, 2015; 197p. Unpublished.

42. Burden, P.L.A.; Davies, C.P.N. Exploration to first production on block 9 off South Africa. Oil Gas J. 1997, 1, 92-98.

43. Davies, C.P.N. Geochemical Correlation of Oils in 14A Reservoirs in Wells E-BT1, EBTO1P, E-AR1, E-AA1 and E-AD1. SOEKOR Unpublished Report, SOE-GCH-RPT-131. 1994; 41p.

44. Dickinson, W.R.; Suczek, C. Plate tectonics and sandstone composition. AAPG Bull. 1979, 63, $2164-2194$.

45. Dickinson, W.R.; Beard, S.; Brakenbridge, F.; Erjavec, J.; Fergusion, R.; Inman, K.; Knepp, R.; Linberg, P.; Ryberg, P. Provenance of the North American Phanerozoic sandstones in relation to tectonic setting. Geol. Soc. Am. Bull. 1983, 64, 222-235. [CrossRef]

46. Rietveld, H.M. The Rietveld method. Phys. Scr. 2014, 89, 1-9. [CrossRef]

47. Dott, R.H. Wackes, greywacke and matrix: What approach to immature sandstone classification. J. Sediment. Petrol. 1964, 34, 625-632.

48. Pettijohn, F.J.; Potter, P.E.; Siever, R. Sand and Sandstones; Springer: New York, NY, USA, 1972; 513p.

49. Pettijohn, F.G.; Potter, P.E.; Siever, R. Sand and Sandstone; Springer: New York, NY, USA, 1973; 618p.

50. Folk, R.L. Petrology of Sedimentary Rock, 2nd ed.; Hemphill Press: Austin, TX, USA, 1974; 182p.

51. Pettijohn, F.J.; Potter, P.E.; Siever, R. Sand and Sandstone; Springer: New York, NY, USA, 1987; 553p.

52. Herron, M.M. Geochemical classification of terrigenous sands and shales from core or log data. J. Sediment. Petrol. 1988, 58, 820-829. 